\title{
Probability Distribution of Low Flows
}

GEOLOGIGAL SURVEY PROFESIIONAL PAPER 434-A

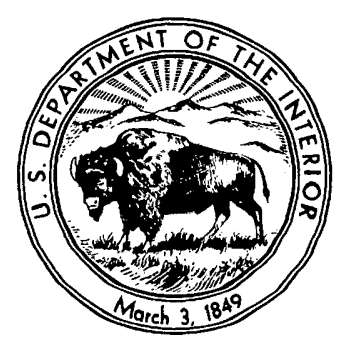




\section{Probability Distribution}

of Low Flows

By NICHOLAS C. MATALAS

S T A T I S T I C A L S T U D I E S I N H Y D R O L O G Y

GEOLOGICAL SURVEY PROFESIONAL PAPER 434-A

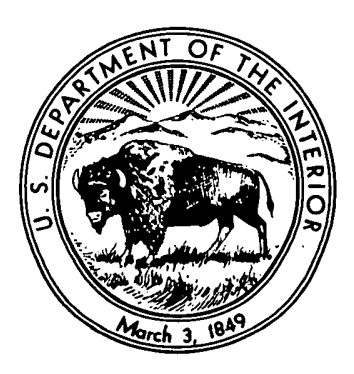

UNITED STATES GOVERNMENT PRINTING OFFICE, WASHINGTON : 1963 


\section{UNITED STATES DEPARTMENT OF THE INTERIOR \\ STEWART L. UDALL, Secretary \\ GEOLOGIGAL SURVEY}

Thomas B. Nolan, Director

For sale by the Superintendent of Documents, U.S. Government Printing Office Washington 25, D.C. 


\section{CONTENTS}

Abstract...

Introduction.

Theoretical probability distributions. . . . . . . . . .

Object of the investigation .........................

Statistical characteristics of low flows . . . . . . . . . . . .

Cumulative probabilities of low flows .............

Skewness and kurtosis of low flows.......

Discussion of probability distributions.......... Characteristics of theoretical distributions . . . . . . . . Gumbel's limited distribution of the smallest value.. 3-Parameter Log-Normal distribution.............. Pearson Type III distribution

Pearson Type $\mathrm{V}$ distribution . . . . . . . . . . . . . Analyses of low flows.

Investigation of the lower limit

Page
A1
1
1
2
2
2
2
2
3
3
3
4
5
6
6
6

Analyses of low flows-Continued

Effect of the largest low flows on the lower limit...-

Investigation of the relation between skewness and kurtosis . . . . . . . . . . . . . .

Method of maximum likelihood applied to low-flow probability analysis ..................................... Discussion of the method of maximum likelihood . . Maximum-likelihood estimates of parameters........ Relative efficiency of moment estimates with respect to maximum-likelihood estimates. ................ Application of the method of maximum likelihood. Summary and conclusions ... . . . . Probability distribution of low flows............ Method of maximum likelihood References

Page

A7

8

9

9

9

9

11

11

11

12

27

\section{ILLUSTRATIONS}

Figures 1-34. Low-flow probability distribution.

1. Middle Branch Westfield River at Goss Heights, Mass _..........

2. Pemigewasset River at Plymouth, N.H.

3. Saco River near Cornish, Maine...-

4. St. John River below Fish River at Fort Kent, Maine.............

5. Souhegan River near Merrimack, N.H

6. Little River near Princeton, N.C

7. Fishing Creek near Enfield, N.C_. -

8. Haw River near Benaja, N.C.....-

9. East Fork of Deep River near High Point, N.C. ...................

10. Neuse River near Clayton, N.C...-

11. Cape Fear River at Lillington, N.C.

12. French Broad River near Asheville, N.C. . . . . . . . . . . . . . . . . .

13. Coeur d'Alene River near Cataldo, Idaho

14. Kootenai River near Leonia, Idaho.

15. Kootenai River at Bonner's Ferry, Idaho .........................

16. Kootenay River near Newgate, British Columbia

17. North F.ork Stillaguamish River near Arlington, Wash...........

18. South Fork Stillaguamish River near Granite Falls, Wash

19. South Fork Skykomish River near Index, Wash...................

20. Sauk River at Sauk, Wash.......

21. James River at Buchanan, Va.....

22. Susquehanna River at Conklin, N.Y...............................

Page

Figures 1-34. Low-flow probability distribution-Con.

23. Little Tennessee River at Judson, N.C.

24. Chenango River near Chenango, N.Y.

15

15

15

16

16

16

16

17

17

17

17

18

18

18

18

19

19

19

19

20

20

25. East Branch Delaware River at Fish's Eddy, N.Y .............

26. West Branch Delaware River at Hales Eddy, N.Y. . . . . . . . .

27. Greenbrier River at Alderson, W. Va. . . . . . . . . . . . . . .

28. Toccoa River near Dial, Ga.....-

29. Sacandago River near Hope, N.Y.

30. Schroon River at River Bank, N.Y

31. South Fork of Holston River near Bluff City, Tenn ..............

32. Brandywine River at Chadd's Ford, $\mathbf{P a}_{\ldots} \ldots \ldots$

33. Hoosic River near Eagle Bridge,

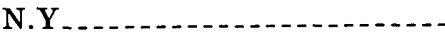

34. West Branch of Oswegatchie River near Harrison, N.Y.............

35. Relation between skewness and standardized lower limits for theoretical probability distributions.................

36. Relation between skewness and kurtosis for theoretical probability distributions.

Page

37-43. Cumulative probability distribution for a random sample from a Pearson Type III distribution. $37,38 . \quad \mathrm{N}=20 \ldots \ldots$ 39-41. $\mathrm{N}=30 \ldots \ldots \ldots$ $42,43 . \quad \mathrm{N}=40 \ldots$ 


\section{TABLES}

Page

TABLE 1. Records of annual minimum daily low flows...

2. Records of annual minimum 7-day low flows..

3. Coefficients of skewness of low-flow data with 90-percent, 95-percent, and 99-percent confidence limits.................................

4. Coefficients of kurtosis of low-flow data with 90-percent, 95-percent, and 99-percent confidence limits............................
A12 TABLE 5. Observed and theoretical standardized lower

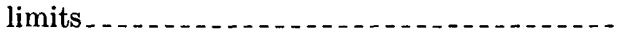
6-8. Relative efficiency of moment estimates with respect to maximum-likelihood estimates. 6. Pearson Type III distributions.......

7. 3-parameter log-normal distributions. -

8. Pearson Type V distributions........

9. Comparison of the methods of maximum likelihood and moments . . . . . .
Page 


\title{
PROBABILITY DISTRIBUTION OF LOW FLOWS
}

\author{
By Nicholas C. Matalas
}

\begin{abstract}
The fit of four theoretical probability distributions to low-flow data was investigated. Also, a study was made to determine the desirability of estimating parameters of probability distributions by the method of maximum likelihood. The four probability distributions used in these investigations were: (1) Gumbel's limited distribution of the smallest value, (2) $3^{-}$ Parameter Log-Normal distribution, (3) Pearson Type III distribution, and (4) Pearson Type V distribution.

The goodness of fit was based on the relation between the observed minimum low flows and the lower limits of the four probability distributions and on the relation between the skewness and kurtosis of the low-flow data. It was found that the Gumbel and Pearson Type III distributions fitted the data equally well and were more representative of the probability distribution of low flows than either the 3-Parameter Log-Normal or Pearson Type V distribution.

The variances of the moment and maximum likelihood estimates of the parameters of probability distributions are functions of the skewness of the data. Based on an a verage skewness for low-flow data of nearly 1 , the variances of moment estimates are nearly twice as large as those of maximum likelihood estimates. Thus future studies dealing with the probability distribution of low flows might best be made by using maximum-likelihood estimates of parameters rather than moment estimates.
\end{abstract}

\section{INTRODUCTION}

\section{THEORETICAL PROBABILITY DISTRIBUTIONS}

A drought is defined, in a broad sense, as an extended period of dryness. In order to define and analyze droughts properly, it is necessary to consider droughts as being two dimensional. These two dimensions are magnitude und duration. In this study, however, only magnitude will be considered.

In an analysis of drought magnitudes, the minimum discharge during each year is considered. The minimum discharge is defined as the average minimum discharge for a given period of time within a year. The given period of time is generally taken to be 1 day, or 7 days if it is necessary to remove the effect of fluctuations resulting from minor river regulation. Other periods of time are used depending upon the problem at hand. These average minimum annual discharges are referred to as low flows. The records of annual minimum daily low flows and minimum 7-day low flows used in this study are listed in tables 1 and 2 , respectively.
For a given stream for which $N$ years of observations are available, $N$ values of low flow can be obtained. These $N$ values are assumed to be a sample from an infinite population of low-flow values. The $N$ values constitute the low-flow record and they are the basis for determining the probability of occurrence of low flows of various magnitudes. This is done by arranging the $N$ values in order of magnitude, largest to smallest, and assigning a cumulative probability or return period (reciprocal of cumulative probability) to each value. The cumulative probability of the $m$ th-order low flow is given by $(N+1-m) /(N+1)$. Plotting the magnitudes of low flow against their respective cumulative probabilities makes possible the construction of a line passing through the plotted points representing the theoretical probability distribution of low flows.

It is assumed that a theoretical probability distribution of low flow exists for each stream. The form of this distribution may be altered if any climatological changes or physical alterations of the drainage basin occur.

An empirical approach to determining the form of the theoretical probability distribution of low flows is to fit several theoretical distributions to observed data and to decide, by suitable criteria, which distribution fits the data best. This approach is facilitated by restricting the number of possible theoretical probability distributions to those which comply with practical statistical and physical considerations.

Because low flows cannot assume values less than 0 , it is necessary that the theoretical probability distributions have lower limits equal to or greater than 0 . Also, it is necessary that the theoretical probability distribution be defined by no more than 3 parameters. This is due to the fact that $N$ is small so that large sampling errors are associated with parameters which are defined by the fourth and higher order moments. Thus the possible choices of probability distributions are restricted to those having lower limits equal to or greater than 0 and defined by no more than 3 parameters.

Several theoretical probability distributions have been used in hydrologic studies. In recent years Gum- 
bel's limited distribution of the smallest value has been advanced as the theoretical probability distribution of low flows. This distribution has a finite lower limit and is defined by three parameters. The Pearson Type III and the Lond-Normal probability distributions have received considerable attention in hydrology. The fact that these distributions have finite lower limits and can be defined by three parameters indicates that they may be applicable to low flows. A fourth theoretical probability distribution which may be applicable to low flows is the Pearson Type V distribution. This distribution has received very little attention in hydrology; however, it has a finite lower limit and can be defined by three parameters.

\section{OBJECT OF THE INVESTIGATION}

The investigation of low flows was undertaken to determine which theoretical probability distribution best fitted observed values of low flows, and to determine the desirability of estimating the parameters of theoretical probability distributions by the method of maximum likelihood.

In order to evaluate the various theoretical probability distributions, two criteria were considered: the relation between the minimum observed low flow and the estimated lower limits of the theoretical probability distributions, and the relation between the skewness and kurtosis of the low-flow data:

With respect to the first criterion, a theoretical probability distribution was considered to be applicable if its estimated lower limit did not exceed the observed minimum low flow during the period of record. Also, it was considered necessary that the estimated lower limit not be negative.

The second criterion was introduced because there is a unique relationship between the skewness and kurtosis for each of the four theoretical probability distributions. From the low-flow data, estimates were made of the skewness and kurtosis. Using the estimated values of skewness, the values of kurtosis were determined from each of the skewness-kurtosis relations and compared with the estimated values of kurtosis. The theoretical probability distribution that provided the best agreement was taken to be the most applicable to low-flow data.

\section{STATISTICAL CHARACTERISTICS OF LOW FLOWS}

\section{CUMULATIVE PROBABILITIES OF LOW FLOWS}

In order to investigate the probability distribution of low flows, it is necessary to assign cumulative probabilities to the ordered data for each stream. It was previously mentioned that these cumulative probabilities are currently determined by $(N+1-m) /(N+1)$ which is the expected cumulative probability of the $m$ th-order value (the order being from largest to smallest) in a sample of $N$ values. However, the cumulative probabilities used in these studies were the median values given by $\mathrm{H}$ : A. Thomas (written communication, 1958) which are defined by

$$
P=\frac{N(3 N+1)-2-m(3 N+1)}{3\left(N^{2}-1\right)} .
$$

Gumbel (1954) has set forth certain criteria to which formulas for estimating cumulative probabilities should adhere. According to Gumbel, the formula (a) must not be dependent upon an initial distribution of the data, (b) must yield values within the range $m / N$ and $(m-1) / N$, (c) must provide values which are equally spaced in time, (d) must be analytically simple, and (e) must converge to 1 and 0 for $m$ equal to 1 and $N$, respectively, as $N$ approaches infinity. Equation 1 satisfies these criteria, as does the formula $(N+1-m) /$ $(N+1)$.

Cumulative probability plots were made for the low flows for each of the 34 streams on normal-arithmetic probability paper. Other types of probability paper can be used. However, the normal-arithmetic probability paper facilitates the analyses better than any other type of paper. The cumulative probability plots are shown in figures 1 through 34 (at end of report).

\section{SKEWNESS AND KORTOSIS OF LOW FLOWS}

Normal-arithmetic probability paper facilitates the use of a normal distribution since a sample from a normal population theoretically should plot as a straight line. An inspection of figures 1 through 34 shows that a linear plot is the exception rather than the rule. The nonlinearity of the plots indicates that the data can not be assumed to be samples from normal populations. However, in order to determine if the nonlinearity of the plots is due to chance, the skewness and kurtosis were determined for each set of low-flow data and tested for significance. For a normal population, the skewness is 0 and the kurtosis is 3 . The estimate of the skewness, $g_{1}$, is given by

$$
g_{1}=\frac{N^{2}}{(N-1)(N-2)} \frac{m_{3}}{S^{3}}
$$

In order to test the kurtosis for significance, it is convenient to express it in a form so that it equals 0 for a normal population. The estimate of the kurtosis, $g_{2}$, is given by

$$
g_{2}=\frac{N^{2}(N+1)}{(N-1)(N-2)(N-3)} \frac{m_{4}}{S^{4}}-\frac{3(N-1)^{2}}{(N-2)(N-3)} .
$$


The parameters $S, m_{3}$, and $m_{4}$ are defined by

$$
\begin{aligned}
& S=\left[\frac{\sum_{i=1}^{N}\left(x_{i}-\bar{x}\right)^{2}}{(N-1)}\right]^{l}, \\
& m_{3}=\frac{1}{N} \sum_{i=1}^{N}\left(x_{i}-\bar{x}\right)^{3},
\end{aligned}
$$

and

respectively, where

$$
m_{4}=\frac{1}{N} \sum_{i=1}^{N}\left(x_{i}-\bar{x}\right)^{4},
$$

$$
\bar{x}=\frac{1}{N} \sum_{i=1}^{N} x_{i}
$$

and $x_{i}$ is the $i$ th observed low_flow. Equations 4 through 7 define the standard deviation; the third central moment, the fourth central moment, and the mean, respectively.

If $g_{1}$ and $g_{2}$ are based on the sample from a normal population, then their respective variances are

$$
V\left(g_{1}\right)=\frac{6}{N+3}
$$

and

$$
V\left(g_{2}\right)=\frac{24}{N+5}
$$

Because of sampling errors, the values of $g_{1}$ and $g_{2}$ may differ from zero even if the data are from a normal population. If

$$
g_{1}<t_{\alpha}\left[\frac{6}{N+3}\right]^{k}
$$

and

$$
g_{2}<t_{\alpha}\left[\frac{24}{N+5}\right]^{k},
$$

where $t_{\alpha}$ is the standard normal deviate corresponding to probability $\alpha$, the data may be regarded as normally distributed with a confidence probability $\alpha$.

The low flows for each of the 34 streams were tested for normality at probability levels of $\alpha$ equal to 0.90 , 0.95 , and 0.99 . For these probability levels, $t_{\alpha}$ equals $1.64,1.96$, and 2.58. This analysis is summarized in tables 3 and 4 . With respect to the skewness, $g_{1}$, the low flows for 19,17 , and 15 streams departed significantly from normality at probability levels 0.90 , 0.95 , and 0.99 , respectively. The kurtosis, however, did not indicate as strong a departure from normality as did the skewness, since the data for only 10, 9, and 7 streams were found to be significantly nonnormal at probability levels $0.90,0.95$, and 0.99 , respectively.

The fact that approximately 50 percent of the streams possess nonnormally distributed data indicates that the low flows can not be considered as samples from normal populations.

\section{DISCUSSION OF PROBABILITY DISTRIBUTIONS Characteristics of theoretical DISTRIBUTIONS}

Theoretical and practical considerations indicate that the probability distribution of low flows is skewed with a lower limit equal to or greater than 0 . The previous analysis of the skewness and kurtosis supports these considerations. Four theoretical probability distributions which are investigated are: (a) Gumbel's limited distribution of the smallest value; (b) the 3-Parameter Log-Normal distribution, (c) Pearson's Type III distribution, and (d) Pearson's Type V distribution. Each of these distributions is skewed, has 3 parameters, and has a lower limit.

\section{GUMBEL'S LIMITED DISTRIBUTION OF THE SMALIEST VALUE}

The limited distribution of the smallest value may be expressed as

$$
\dot{p}(x)=\frac{\alpha}{u-\epsilon}\left(\frac{x-\epsilon}{u-\epsilon}\right)^{\alpha-1} \exp -\left[\left(\frac{x-\epsilon}{u-\epsilon}\right)^{\alpha}\right] ; \quad \epsilon \leq x<\infty,
$$

wherein $\epsilon$ denotes the lower limit, $u$ denotes the characteristic drought ( $u$ is the value of $x$ which will be exceeded 36.788 percent of the time), and $\alpha$ is a scale parameter (analogous to the standard deviation, $s$, for a normal probability distribution). In order to fit the Gumbel distribution to a set of observations, it is necessary to estimate $\epsilon, u$, and $\alpha$. These estimates are functions of $\bar{x}, s$, and $m_{3}$.

By using the method of moments (Gumbel, 1954), the mean, $\mu_{1}^{1}$, is defined as

$$
\mu_{1}^{1}=\dot{\epsilon}+(u-\epsilon) \Gamma(1+1 / \alpha) .
$$

The second, third, and fourth central moments are given by

$$
\begin{aligned}
& \mu_{2}=(u-\epsilon)^{2}\left[\Gamma(1+2 / \alpha)-\Gamma^{2}(1+1 / \alpha)\right] \\
& \mu_{3}=(u-\epsilon)^{3}[\Gamma(1+3 / \alpha)-3 \Gamma(1+2 / \alpha) \Gamma(1+1 / \alpha) \\
& \left.+2 \Gamma^{3}(1+1 / \alpha)\right], \\
& \text { and } \\
& \begin{aligned}
\mu_{4}=(u-\epsilon)^{4}[\Gamma(1+4 / \alpha)-4 \Gamma(1+3 / \alpha) \Gamma(1+1 / \alpha) \\
\left.+6 \Gamma(1+2 / \alpha) \Gamma^{2}(1+1 / \alpha)-3 \Gamma^{4}(1+1 / \alpha)\right]
\end{aligned}
\end{aligned}
$$

respectively. $\Gamma(\quad)$ denotes the gamma function of the argument within the parentheses. 
The skewness and kurtosis are defined respectively as

and

$$
G_{1}=\frac{\mu_{3}}{\mu_{3}^{3 / 2}}=\frac{\left[\Gamma(1+3 / \alpha)-3 \Gamma(1+2 / \alpha) \Gamma(1+1 / \alpha)+2 \Gamma^{3}(1+1 / \alpha)\right]}{\left[\Gamma(1+2 / \alpha)-\Gamma^{2}(1+1 / \alpha)\right]^{3 / 2}}
$$

$$
G_{2}=\frac{\mu_{4}}{\mu_{2}^{2}}-3=\frac{\left[\Gamma(1+4 / \alpha)-4 \Gamma(1+3 / \alpha) \Gamma(1+1 / \alpha)+6 \Gamma(1+2 / \alpha) \Gamma^{2}(1+1 / \alpha)-3 \Gamma^{4}(1+1 / \alpha)\right]}{\left[\Gamma(1+2 / \alpha)-\Gamma^{2}(1+1 / \alpha)\right]^{2}}-3 .
$$

$G_{1}$ and $G_{2}$ are used to denote the population values of skewness and kurtosis, respectively, to distinguish them from their sample estimates $g_{1}$ and $g_{2}$ given by equations 2 and 3 , respectively.

Equations 17 and 18 show that the skewness and kurtosis are functions of $\alpha$. Hence, if $G_{1}$ or $G_{2}$ is replaced by its respective sample estimate, $g_{1}$ or $g_{2}$, a trial-and-error solution gives the value of $\alpha$. Gumbal (1954b) has given a table prepared by G. R. Garabedian for the solution of equation 17. With $\alpha$ known, $\epsilon$ and $u$ are determined by solving equations 13 and 14 simultaneously; whereby,

$$
u=\mu_{1}^{1}+\mu_{2}^{\frac{1}{2}}[1-\Gamma(1+1 / \alpha)]\left[\Gamma(1+2 / \alpha)-\Gamma^{2}(1+1 / \alpha)\right]^{-\frac{1}{2}}
$$

and

$$
\epsilon=\mu_{1}^{1}+\mu_{2}^{\frac{1}{2}}[\Gamma(1+1 / \alpha)]\left[\Gamma(1+2 / \alpha)-\Gamma^{2}(1+1 / \alpha)\right]^{-\frac{1}{2}} .
$$

In order to determine $u$ and $\epsilon$, it is necessary to replace $\mu_{1}^{1}$ and $\mu_{2}^{\frac{1}{2}}$ by their sample estimates $\bar{x}$ (eq 7) and $S$ (eq 4), respectively. It should be noted that the sample estimates of $\mu_{3}$ and $\mu_{4}$ are $m_{3}$ (eq 5) and $m_{4}$ (eq 6), respectively.

For purposes of curve fitting, equation 12 is expressed as

$$
P(x)=\exp \left[-\left(\frac{x-\epsilon}{u-\epsilon}\right)^{\alpha}\right]
$$

Equation 21 defines the cumulative probability of $x$ obtained by integrating equation 12 from $\epsilon$ to some arbitrary value of $x$. By letting

$$
y=\left(\frac{x-\epsilon}{u-\epsilon}\right)^{\alpha}
$$

equation 21 becomes

$$
P(x) \equiv P(y)=e^{-\nu} .
$$

Thus by using preassigned values of $P(x)$, the corresponding values of $y$ are determined by equation 23 . Knowing $y$ and using the estimates of $\epsilon$, $u$, and $\alpha$, values of $x$ corresponding to the preassigned values of $P(x)$ are determined by equation 22 . This procedure was applied to the low-flow data for each of the 34 streams. The fitted curves are shown in figures 1 through 34 .
Gumbel (1954b) has pointed out that if the data are plotted on logarithmic extremal probability paper, a straight line can be fitted to the data only if $\epsilon=0$, providing, of course, that the data are in accord with the theory. If $\epsilon>0$, the fitted curve is concave downward, and if $\epsilon<0$, the curve is concave upward. However, in the case of low flows, $\epsilon$ must be equal to or greater than 0.

\section{3-PARAMETER LOG-NORMAL DISTRIBUTION}

3-Parameter Log-Normal distribution may be expressed as

$$
p(x)=\frac{1}{\sqrt{2 \pi} \sigma(x-a)} \exp \left\{-\frac{1}{2 \sigma^{2}}[\ln (x-a)-m]^{2}\right\},
$$

where $m$ and $\sigma$ denote the mean and standard deviation, respectively, of the values of $\ln (x-a)$, and $a$ denotes the lower limit. Using the method of moments, the mean is given by

$$
\mu_{1}^{1}=a+\exp \left[\frac{1}{2} \sigma^{2}+m\right],
$$

and the second, third, and fourth central moments are given by

$$
\begin{gathered}
\mu_{2}=e^{2 m} \dot{\gamma}^{2}\left(\gamma^{2}+1\right), \\
\mu_{3}=e^{3 m} \gamma^{4}\left(\gamma^{2}+3\right)\left(\gamma^{2}+1\right)^{3 / 2},
\end{gathered}
$$

and

$$
\mu_{4}=e^{4 m} \gamma^{4}\left(\gamma^{2}+1\right)^{2}\left[\gamma^{8}+6 \gamma^{6}+15 \gamma^{4}+16 \gamma^{2}+3\right],
$$

respectively, wherein

$$
\gamma^{2}=e^{\sigma^{2}}-1
$$

The parameter $\gamma$ is related to the coefficient of variation, $C V$, which is defined as the ratio of the standard deviation to the mean. Thus

$$
C V=\frac{\mu_{2}^{1 / 2}}{\mu_{1}^{1}}=\frac{\gamma}{1+\frac{a}{e^{m}\left(\gamma^{2}+1\right)^{\frac{1}{3}}}} .
$$

If $a=0$, then $C V=\gamma$. 
The skewness, $G_{1}$, and the kurtosis, $G_{2}$, are given by

$$
G_{1}=\frac{\mu_{3}}{\mu_{2}^{3 / 2}}=\gamma^{3}+3 \gamma
$$

and

$$
G_{2}=\frac{\mu_{4}}{\mu_{2}^{2}}-3=\gamma^{2}\left(\gamma^{6}+6 \gamma^{4}+15 \gamma^{6}+16\right) .
$$

If either $G_{1}$ or $G_{2}$ is replaced with its sample estimate $g_{1}$ or $g_{2}, \gamma$ may be obtained by a trial-and-error solution.

With $\gamma$ known, $a$ and $m$ are determined by solving equations 25 and 26 simultaneously; whereby,

$$
a=\mu_{1}^{1}-\frac{\mu_{2}^{1 / 2}}{\gamma}
$$

and

$$
m=\ln \left(\frac{\mu_{2}^{1 / 2}}{\gamma}\right)-\frac{1}{2} \ln \left(\gamma^{2}+1\right) .
$$

Estimates of $a$ and $m$ are obtained by replacing $\mu_{1}^{1}$ and $\mu_{2}^{1 / 2}$ by their sample estimates $\bar{x}$ and $s$, respectively. The parameter $\sigma$ is estimated by

$$
\sigma=\left[\ln \left(\gamma^{2}+1\right)\right]^{1 / 2} .
$$

For purposes of curve fitting, the transformation

$$
z=\frac{1}{\sigma}[\ln (x-a)-m]
$$

is introduced in equation 24; whereby, the cumulative probability, $P$, of $x$ is defined as

$$
P(x) \equiv P(z)=\frac{1}{\sqrt{2 \pi}} \int_{-\alpha}^{z} \exp \left[-\frac{1}{2} z^{2}\right] d_{z},
$$

which is identical to the standardized form of the normal distribution. From tables for the standardized normal distribution, values of $z$ (often denoted by $t$ ) are obtained for preassigned values of $P(z)$. Using these values of $z$ in equation 36 , the corresponding values of $x$ are obtained.

\section{PEARSON TYPE III DISTRIBUTION}

The Pearson Type III distribution can be expressed in various ways. A representation of this distribution, which is well adapted to mathematical treatment, is

$$
p(x)=\frac{1}{a \Gamma(b+1)}\left(\frac{x-m}{a}\right)^{b} \exp \left[-\left(\frac{x-m}{a}\right)\right]
$$

where $a, m$, and $b$ are parameters. By using the method of moments, the mean is defined as

$$
\mu_{1}^{1}=m+a(b+1),
$$

and the second, third, and fourth central moments are

and

$$
\begin{gathered}
\mu_{2}=a^{2}(b+1), \\
\mu_{3}=2 a^{3}(b+1),
\end{gathered}
$$

respectively.

$$
\mu_{4}=3 a^{4}(b+3)(b+4),
$$

The skewness, $G_{1}$, and the kurtosis, $G_{2}$, are given by

and

$$
G_{1}=\frac{\mu_{3}}{\mu_{2}^{3 / 2}}=\frac{2}{(b+1)^{\frac{1}{2}}}
$$

$$
G_{2}=\frac{\mu_{4}}{\mu_{2}^{2}}-3=\frac{6}{b+1} \text {. }
$$

The skewness and kurtosis are both functions of the parameter $b$. Thus by replacing either $G_{1}$ or $G_{2}$ by its sample estimate $g_{1}$ or $g_{2}, b$ may be determined. With $b$ known, the parameters $m$ and $a$ can be determined by the simultaneous solution of equations 39 and 40 This solution gives

and

$$
m=\mu_{1}^{1}-\mu_{2}^{\frac{3}{3}}(b+1)^{\frac{1}{2}}
$$

$$
a=\mu_{2}^{\frac{3}{3}}(b+1)^{-\frac{1}{2}} \text {. }
$$

Estimates of $m$ and $a$ are obtained by replacing $\mu_{1}^{1}$ and $\mu_{2}^{z}$ by their sample estimates $\bar{x}$ and $s$, respectively.

If the transformations

$$
\begin{aligned}
& t=\frac{x-\bar{x}}{s}, \\
& b+1=A^{2},
\end{aligned}
$$

and

$$
u=A(t+A)
$$

are introduced into equation 38 , the cumulative probabilities are defined by

$$
P(x)=P(u)=\frac{1}{\Gamma\left(A^{2}\right)} \int_{0}^{u} e^{-u} u^{A^{2}-1} d u
$$

where $2 u$ is distributed as chi-square with $2 A^{2}$ degrees of freedom. From a table of the chi-square distribution, values of $2 u$ are obtained corresponding to $2 A^{2}$ degrees of freedom for preassigned cumulative probabilities. By using equation 49, values of $t$ are obtained corresponding to the values of $u$. Values of $x$ are obtained corresponding to the $t$ values by equation 47 . In this manner, the Pearson Type III distribution was fitted to the low-flow data for each of the 34 streams. The fitted curves are shown in figures 1 through 34 . 


\section{PEARSON TYPE V DISTRIBUTION}

The Pearson Type V distribution may be expressed as

$$
p(x)=\frac{1}{a \Gamma(b-1)}\left(\frac{x-m}{a}\right)^{-b} \exp \left[-\left(\frac{a}{x-m}\right)\right]
$$

where $m, a$, and $b$ are parameters. The Pearson Type III distribution involves the term $(x-m) / a$, whereas the Pearson Type V distribution involves the reciprocal of this term.

By using the method of moments, the mean is defined as

$$
\mu_{1}^{1}=m+\frac{a}{b+2}
$$

and the second, third, and fourth central moments are given by

and

$$
\begin{gathered}
\mu_{2}=a^{2}(b-2)^{-2}(b-3)^{-1}, \\
\mu_{3}=4 a^{3}(b-2)^{-3}[(b-3)(b-4)]^{-1},
\end{gathered}
$$

$$
\mu_{4}=3 a^{4}(b+4)(b-2)^{-4}[(b-3)(b-4)(b-5)]^{-1},
$$

respectively.

The skewness, $G_{1}$, and the kurtosis, $G_{2}$, are defined as

$$
G_{1}=\frac{\mu_{3}}{\mu_{2}^{3 / 2}}=\frac{4(b-3)^{\frac{1}{2}}}{(b-4)}
$$

and

$$
G_{2}=\frac{\mu_{4}}{\mu_{2}^{2}}-3=\frac{6(5 b-16)}{(b-4)(b-5)}
$$

The skewness and kurtosis are both functions of the parameter $b$. If either $G_{1}$ or $G_{2}$ is replaced by its same estimate $g_{1}$ or $g_{2}$, the parameter $b$ can be determined.

With $b$ known, the parameters $m$ and $a$ may be determined by the simultaneous solution of equations 52 and 53. This solution gives

and

$$
m=\mu_{1}^{1}-\mu_{2}^{1 / 2}(b-3)^{\frac{1}{2}}
$$

$$
a=\mu_{2}^{1 / 2}(b-2)(b-3)^{\frac{1}{2}} .
$$

Estimates of $m$ and $a$ may be obtained by replacing $\mu_{1}^{1}$ and $\mu_{2}^{\frac{1}{3}}$ by their sample estimates $\bar{x}$ and $s$, respectively.

If the transformation

$$
u=\left[(b-3)^{\frac{1}{3}}+\left(\frac{x-\bar{x}}{s}\right)\right]^{-1}(b-2)(b-3)^{\frac{1}{2}}
$$

is introduced into equation 51 , the cumulative probabilities are defined by

$$
P(x)=P(u)=\frac{1}{\Gamma(b-1)} \int_{0}^{u} u^{b-2} e^{-u} d u,
$$

where $2 u$ is distributed as chi-square with $2(b-1)$ degrees of freedom. By means of a table of the chisquare distribution, values of $2 u$ corresponding to preassigned values of $P(u)$ can be obtained; whereby, the corresponding values of $x$ are obtained by equation 60. This procedure may be used to fit a Pearson Type $\mathrm{V}$ distribution to a set of data.

\section{ANALYSES OF LOW FLOWS}

\section{INVESTIGATION OF THE LOWER LIMIT}

The first criterion given for the applicability of a theoretical probability distribution was that its lower limit be positive and not exceed the minimum observed low flow. A theoretical probability distribution underestimates the severity of the extreme droughts if its lower limit is greater than the minimum observed low flow.

In order to facilitate the investigation of the lower limit, the lower limit was expressed as a standardized variate in the form

$$
t=\frac{x_{\min }-\mu_{1}^{1}}{\mu_{2}^{1 / 2}}
$$

where $x_{m i n}$ denotes the minimum value or lower limit. By using the appropriate values of $x_{m i n}, u_{1}^{1}$, and $u_{2}^{\frac{1}{2}}$, previously given for each of the theoretical probability distributions, the standardized lower limits for the Gumbel, 3-Parameter Log-Normal, Pearson Type III, and Pearson Type V distributions are given by

$$
\begin{gathered}
t_{G}=-[\Gamma(1+1 / \alpha)]\left[\Gamma(1+2 / \alpha)-\Gamma^{2}(1+1 / \alpha)\right]^{-\frac{1}{1}}, \\
t_{L N}=-\frac{1}{\gamma} \\
t_{I I I}=-(b+1)^{\frac{1}{2}}
\end{gathered}
$$

and

$$
t_{V}=-(b-3)^{\frac{1}{2}},
$$

respectively.

It is interesting to note that, for each theoretical probability distribution, the standardized lower limit is a function of a single parameter which defines the skewness. In figure 35 , the relation between the absolute value of the standardized lower limit and the skewness is shown for each of the four theoretical probability distributions. Figure 35 shows that for a given value of skewness, $t_{V}<t_{L N}$ and that $t_{L N}<t_{G}$ or $t_{I I J}$. For values of skewness greater than $2, t_{I I I}<t_{\sigma}$, and for values of skewness within the range 0.35 to 2 , $t_{G}<t_{I I I}$, and for values of skewness less than $0.35, t_{I I I}<t_{\sigma}$.

In table 5, the standardized lower limits for each of the theoretical probability distributions are given for each of the 34 streams. These values are based on the 
estimates of $x_{m i n}, \mu_{1}^{1}$, and $\mu_{2}^{\frac{1}{t}}$. The observed minimum low flow for each stream expressed in standardized form, $t_{0}$, is given in table 5. Also, the standardized lower limits, denoted by $t^{\prime}$, are given in table 5 , based on the assumption that the observed minimum low flow is 0 for each of the 34 streams.

From table 5, it is seen that 10 and 15 streams have experienced minimum discharges less than the estimated lower limits for the Gumbel and Pearson Type III distributions, respectively. The estimated lower limits for the 3-Parameter Log-Normal and Pearson Type $\mathrm{V}$ distributions are less than the observed minimum discharge for each of the 34 streams. The estimated lower limits for each of the theoretical probability distributions yield negative values of discharge if they are less than their corresponding values of $t^{\prime}$. From table 5 , it is seen that for $3,4,21$, and 34 streams, the estimated lower limits for the Gumbel, Pearson Type III, 3-Parameter Log-Normal, and Pearson Type V distributions, respectively, yield negative values of discharge for the lower limit.

The above investigation of the lower limit shows that the Gumbel and Pearson Type III distributions are more applicable to low flows than either the 3Parameter Log-Normal or Pearson Type V distributions, since these latter 2 distributions, although having lower limits smaller than the observed minimum low flows for the 34 streams, yield large negative values of discharge for the lower limits.

\section{EFFECT OF THE LARGEST LOW FLOWS ON THE LOWER LIMIT}

By inspecting the cumulative probability plots of the low-flow data for the 34 streams shown in figures 1 through 34 , it is seen that the largest low flow often appears to deviate appreciably from the second largest low flow with respect to the deviations between the smaller low flows. In the estimation of the skewness (eq 2), the third central moment, $m_{3}$, which involves the cube of the departures of the low flows from their mean, can be very large if the largest low flow deviotes appreciably from the second largest low flow. Since the lower limit is a function of the skewness, such deviations can lead to large values of skewness, which, in turn, can lead to the theoretical distribution underestimating the severity of the extreme droughts.

This situation is shown by the low-flow data for the Kootenai River at Leonia, Idaho (fig. 14). By neglecting the largest low flow, the data plot very nearly along a straight line on normal-arithmetic probability paper, indicating that the skewness is nearly zero. However, the inclusion of the largest low flow yields an estimate of skewness of 1.85 which differs significantly from 0 at the 99-percent level. The magnitude of the smallest low flow is 1,070 cfs (cubic feet per second). The lower limits based on the Gumbel and the Pearson Type III distributions are 1,370 cfs and 1,372 cfs, respectively. Thus both theoretical probability distributions underestimate the severity of the extreme droughts.

A tendency for the largest low flow to deviate excessively from the other low flows was observed in the data for several of the streams. With respect to the Kootenai River at Bonners Ferry, Idaho, Little Tennessee River at Judson, N.C., South Fork of the Holston River near Bluff City, Tenn., and West Branch of the Oswegatchie River near Harrisville, N.Y., the largest low flow deviated sufficiently from the second largest low flow so that both the Gumbel and Pearson Type III distributions yielded lower limits greater than the observed minimum low flow. For the St. John River below Fish River at Ft. Kent, Maine, Coeur d'Alene River near Cataldo, Idaho, and Kootenay River at Newgate, British Columbia, the deviation between the largest and the second largest low flow was sufficient to reject the Pearson Type III distribution.

Excessive deviations were not confined to those between the largest and second largest low flows. With respect to the low-flow data for the Schroon River at River Bank, N.Y., the two largest low flows deviated sufficiently from the other low flows to yield lower limits for the Gumbel and Pearson Type III distributions greater than the observed minimum low flow.

Thus, by comparing the cumulative probability plots shown in figures 1 through 34 and the lower limits given in table 5, it is seen that in those cases where the Gumbel and (or) the Pearson Type III distributions were found to be inapplicable, the low-flow data were characterized by the fact that the larger low flows deviated excessively from the other low flows. The question now arises as to whether or not these deviations are due to chance.

In order to answer this question, it is necessary to consider the deviations of the larger values from the other values which arise in random sampling from a Gumbel and Pearson Type III distribution. Within the probability ranges which arise due to the sample sizes of low-flow data, the Gumbel and Pearson Type III distributions are nearly coincident. But because of the mathematical simplicity of the Pearson Type III distribution, the deviations of the larger values from the other values are analyzed on the basis of random sampling from a Pearson Type III distribution.

Pearson Type III distributions with skewness of 1 (average skewness for the 34 streams), mean of 0 , and variance of 1 were plotted on normal-arithmetic probability paper (see figs. 37-43, at end of report). From a table of random rectangular numbers, 2 sets of 20,3 
sets of 30 , and 2 sets of 40 three-digit numbers were randomly selected. These numbers were considered as probabilities expressed in thousandths. The random numbers in each set were arranged in increasing order of magnitude, and the probability corresponding to each order of magnitude was determined by equation 1 . For each probability determined by equation 1, the corresponding value of $t$ was determined graphically from the Pearson Type III distribution. The values of $t$ were then plotted against the randomly selected probabilities corresponding to the probabilities determined by equation 1 .

In figures 37 through 43 , it is seen that the $t$ values plotted against their respective randomly selected probabilities cluster around the Pearson Type III distribution. With respect to each of the sample sizes, a plot was found where the largest value deviated excessively from the other values. As the sample size increases, these deviations tend to decrease. Although the random sampling technique does not answer the question conclusively as to whether or not the deviations are attributable to chance, it does support the notion that the deviations are due to chance. Therefore, the $N$ values of low flow taken from $N$ years of record may be regarded as belonging to the same distribution. This conclusion, of course, does not preclude the possibility that the deviations are significant and can be attributed to physical considerations of the low flows. However, such a position is difficult to prove due to the large sampling errors.

\section{INVESTIGATION OF THE RELATION BETWEEN SKEWNESS AND KURTOSIS}

The second criterion considered in selecting the lowflow probability distribution was the agreement between the estimated values of skewness and kurtosis and the theoretical relations between skewness and kurtosis for each of the four theoretical probability distributions. For each of the four theoretical probability distributions, there exists a unique relationship between skewness and kurtosis. From the low flow data, estimates of skewness and kurtosis were determined by equations 2 and 3, respectively. By using the estimated values of skewness, the kurtosis was determined from each of the four theoretical relationships of skewness and kurtosis. These values of kurtosis were then compared with the values of kurtosis estimated by equation 3 .

The relation between skewness and kurtosis was expressed by the parameters $\beta_{1}$ and $\beta_{2}$, which were defined as

and

$$
\beta_{1}=G_{1}^{2}=\mu_{3}^{2} / \mu_{2}^{3}
$$

$$
\beta_{2}=G_{2}+3=\mu_{4} / \mu_{2}^{2}
$$

From the previously defined expressions for $G_{1}$ and $G_{2}$ for each of the four theoretical probability distributions, the relations between $\beta_{1}$ and $\beta_{2}$ were determined. These relations are shown in figure 36 . From figure 36, it is seen that for each of the four theoretical probability distributions, the kurtosis increases monotonically with an increase in skewness. Except for the Gumbel distribution, the kurtosis, $\beta_{2}$, is 3 when the skewness, $\beta_{1}$, is 0 . Since a skewness equal to 0 and a kurtosis equal to 3 uniquely define the normal probability distribution, then, as the skewness approaches 0 , the 3-Parameter Log-Normal; the Pearson Type III, and the Pearson Type V distributions converge asymptotically to the normal distribution.

From figure 36 , it is seen that for a given value of skewness, the kurtosis of the Pearson Type $V$ distribution is greater than that of the 3-Parameter Log-Normal distribution. Also, for a given value of skewness, the kurtosis of the 3-Parameter Log-Normal distribution is greater than that of the Gumbel and the Pearson Type III distributions. For values of $\beta_{1}$ in the range 0 to 4 , the kurtosis of the Pearson Type III distribution is greater than that of the Gumbel distribution, and, for values of $\beta_{1}$ greater than 4 , the reverse is true.

Estimates of $\beta_{1}$ and $\beta_{2}$, denoted by $\hat{\beta}_{1}$ and $\hat{\beta}_{2}$, respecgively, were obtained for the low-flow data for each of the 34 streams by means of

$$
\hat{\beta}_{1}=g_{1}^{2} .
$$

and

$$
\hat{\beta}_{2}=g_{2}+\frac{3(N-1)^{2}}{(N-2)(N-3)} .
$$

The values of $g_{1}$ and $g_{2}$ are given by equations 2 and 3 , respectively. The estimated values $\hat{\beta}_{1}$ and $\hat{\beta}_{2}$ are shown as circles in figure 36 . By inspecting figure 36 , it is seen that the values $\hat{\beta}_{1}$ and $\hat{\beta}_{2}$ fail to cluster around any one of the $\beta_{1}$ vs. $\beta_{2}$ relations for the 4 theoretical probability distributions. For values of $\hat{\beta}_{1}$ in the range 0 to 1 , the values of $\hat{\beta}_{1}$ and $\hat{\beta}_{2}$ are dispersed around each of the $\beta_{1}$ vs. $\beta_{2}$ lines of relation. However, for values of $\beta_{1}>1$, nearly all the values of $\hat{\beta}_{1}$ and $\hat{\beta}_{2}$ plot above the Gumbel and Pearson Type III $\beta_{1}$ vs. $\beta_{2}$ lines of relation and consequently above the 3-Parameter Log-Normal and the Pearson Type $V \beta_{1}$ vs. $\beta_{2}$ lines of relation. Therefore, for small values of $\beta_{1}$, each of the 4 theoretical probability distributions can be used to represent the data; whereas, for values of $\beta_{1}>1$, each of the 4 theoretical probability distributions fails to represent the data adequately. However, within the total range of the observed values of $\beta_{1}$ and $\beta_{2}$, the Gumbel and the Pearson Type III distributions are more representative 
of the distribution of low flows than the 3-Parameter Log-Normal and the Pearson Type V distribution.

The mean values of $\hat{\beta}_{1}$ and $\hat{\beta}_{2}$ were found to be 1.23 and 4.27 , respectively. These values are plotted on the graph of the $\beta_{1}$ vs. $\beta_{2}$ relations (fig. 36). The mean values of $\hat{\beta}_{1}$ and $\hat{\beta}_{2}$ plot above the Gumbel line.

The investigation of the $\beta_{1}$ vs. $\beta_{2}$ relations indicates that the Gumbel and the Pearson Type III distributions fit the observed low-flow data better than the 3-Parameter Log-Normal or the Pearson Type V distributions. Thus, both the first and second criteria indicate that the Gumbel and the Pearson Type III distributions are more representative of the probability distribution of low flows than the 3-Parameter Log-Normal and Pearson Type V distributions.

\section{METHOD OF MAXIMUM LIKELIHOOD APPLIED TO LOW-FLOW PROBABILITY ANALYSIS}

\section{DISCUSSION OF THE METHOD OF MAXIMUM} IIKELIHOOD

The preceding investigations of the probability distribution of low flows were based on the classical method of moments. These investigations were based on the comparison among various statistical parameters which were determined by the method of moments. An alternate method of determining the parameters is that of maximum likelihood. This method yields the most efficient estimate of a parameter when such an estimate exists. The most efficient estimate of a parameter is defined as that estimate which has the minimum variance. The most efficient estimate is not, however, always an unbiased estimate (Hoel, 1954). However, a biased estimate which has minimum variance may be preferred to an unbiased estimate which has a large variance. A property of the method of maximum likelihood which recommends it for low-flow probability analysis is that the lower limit of a theoretical probability distribution can never exceed the minimum observed value.

The investigation of the method of maximum likelihood was undertaken to determine its usefulness in studying the probability distribution of low flows. This investigation was carried out by comparing the variances of the maximum-likelihood estimates with the variances of the moment estimates of the parameters of the theoretical probability distributions.

\section{MAXIMUM-LIKELIHOOD ESTIMATES OF PARAMETERS}

The probability distribution of a random variable $x$ may be expressed as

$$
d p(x)=f\left(a_{1}, a_{2}, \ldots, a_{k}: x\right) d x,
$$

where $a_{1}, a_{2}, \ldots, a_{k}$ are the parameters of the distribution. The likelihood function, defined as the natural logarithm of the probability function summed over all possible values of $x$, is given by

$$
L=\sum_{1}^{N} \ln \left[f\left(a_{1}, a_{2}, \ldots, a_{k}: x\right)\right]
$$

The maximum-likelihood estimates of the parameters $a_{1}, a_{2}, \ldots, a_{k}$ are those estimates which minimize the likelihood function, $L$. These estimates are obtained by setting the partial derivatives of $L$ with respect to each parameter equal to 0 and solving the $k$ simultaneous equations. These equations are generally nonlinear and must be solved by some iterative process.

The variances and the mean cross products (covariances) of these parameters are obtained from the expected values of the second partial derivatives of $L$ with respect to the parameters. If $\partial^{2} L / \partial a_{i}^{2}$ and $\partial^{2} L / \partial a_{i} \partial a$, denote the second partial derivates of $L$ with respect to $a_{i}$ and $a_{i} a_{j}(i \neq j)$, respectively, the Hessian determinant is

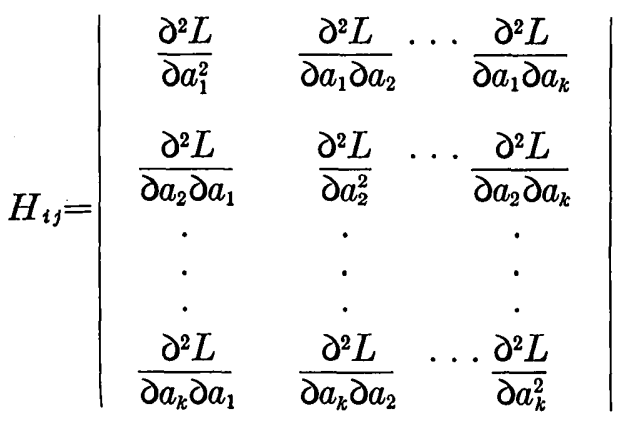

In order to obtain the variance of $a_{i}$, the minor of the Hessian determinant corresponding to the $i$ th parameter is divided by $H_{i j}$. Thus

$$
V\left(a_{i}\right)=\frac{\text { Minor }\left(\partial^{2} L / \partial a_{i}^{2}\right)}{H_{i j}} .
$$

Similarly, the covariance of $a_{\mathfrak{t}}$ and $a_{f}$ is given by

$$
C\left(a_{i} a_{j}\right)=\frac{\text { Minor }\left(\partial^{2} L / \partial a_{i} \partial a_{j}\right)}{H_{i j}} .
$$

RELATIVE EFFICIENCY OF MOMENT ESTIMATES
WITH RESPECT TO MAXIMUM-LIKELIHOOD ESTIMATES

The variances of the moment estimates of $a_{1}, a_{2}, \ldots$, $a_{k}$ may be obtained by the method of variation. If $V\left[M L\left(a_{i}\right)\right]$ and $V\left[M\left(a_{i}\right)\right]$ denote the variances of the maximum likelihood and moment estimates of $a_{i}$, respectively, then the relative efficiency, $E$, (Fisher, 1922) between the two methods with respect to $a_{t}$ is given by

$$
E\left(a_{i}\right)=\frac{V\left[M L\left(a_{i}\right)\right]}{V\left[M\left(a_{i}\right)\right]} .
$$


Since the method of maximum likelihood yields the estimate of a parameter with minimum variance, then $0 \leq E\left(a_{i}\right) \leq 1$. If the variable $x$ is normally distributed, the method of maximum likelihood is identical to the method of moments. In this case $E\left(a_{i}\right)=1$.

The relative efficiencies are given for the parameters defining the Pearson Type III, the 3-Parameter LogNormal, and the Pearson Type V distributions. The Gumbel distribution is not treated since $\partial^{2} L / \partial \epsilon^{2}$ can not be defined for $\alpha<2$.

For the Pearson Type III distribution (eq 38), the relative efficiencies with respect to the parameters $m$, $a$, and $b$ are

$$
E(m)=1-\frac{2}{b+1}
$$

$$
\begin{gathered}
E(a)=\frac{\ln \left(\gamma^{2}+1\right)}{\gamma^{2}\left(\gamma^{2}+1\right)^{3}\left[\ln \left(\gamma^{2}+1\right)+1\right]}, \\
E(m)=\frac{\ln \left(\gamma^{2}+1\right)\left[\ln \left(\gamma^{2}+1\right)+1\right]\left(\gamma^{2}+1\right)}{\left\{\left[\ln \left(\gamma^{2}+1\right)+1\right]\left(\gamma^{2}+1\right)-1\right\}\left[\left(\gamma^{2}+1\right)^{4}+2\left(\gamma^{2}+1\right)^{3}+3\left(\gamma^{2}+1\right)^{2}-4\right]},
\end{gathered}
$$

and

$$
\begin{aligned}
& E(\sigma)= \\
& \frac{\left\{\begin{array}{c}
\left\{3 6 \{ [ \operatorname { l n } ( \gamma ^ { 2 } + 1 ) ] ( \gamma ^ { 2 } + 1 ) + \gamma ^ { 2 } \} \{ [ \operatorname { l n } ( \gamma ^ { 2 } + 1 ) ] ^ { 2 } ( \gamma ^ { 2 } + 1 ) ^ { 4 } \} \left\{\gamma^{2}\left(\gamma^{2}+1\right)^{15}-12\left(\gamma^{2}+1\right)^{13}+36\left(\gamma^{2}+1\right)^{11}-\left(6 \gamma^{2}+24\right)\left(\gamma^{2}+1\right)^{10}\right.\right. \\
+60\left(\gamma^{2}+1\right)^{9}-180\left(\gamma^{2}+1\right)^{7}+15 \gamma^{2}\left(\gamma^{2}+1\right)^{6}-\left[24 \gamma^{8}-9\left(\gamma^{3}+3 \gamma\right)^{2}-120\right]\left(\gamma^{2}+1\right)^{4}-\left[48 \gamma^{8}-18\left(\gamma^{3}+3 \gamma\right)^{2} \gamma^{8}+20 \gamma^{2}\right. \\
\left.+288]\left(\gamma^{2}+1\right)^{3}-\left[72 \gamma^{8}-27\left(\gamma^{3}+3 \gamma\right)^{2} \gamma^{8}+360\right]\left(\gamma^{2}+1\right)^{2}+\left(15 \gamma^{2}+384\right)\left(\gamma^{2}+1\right)-5 \gamma^{2}+72 \gamma^{8}+8\left(\gamma^{3}+3 \gamma\right)^{2} \gamma^{8}+8\right\}
\end{array}\right\}}{\gamma^{2}\left\{\left[\ln \left(\gamma^{2}+1\right)\right]\left(\gamma^{2}-1\right)+\gamma^{2}\right\}} .
\end{aligned}
$$

The relative efficiency of each parameter is a function of $\gamma$, which itself is a function of the skewness. The relative efficiencies of each parameter for various values of skewness are given in table 7.
For the Pearson Type V distribution (eq 51), the relative efficiencies of the parameters $m, a$, and $b$ are given by

$$
\begin{gathered}
E(m)=\frac{(b-2)^{2}(b-3)}{b(b-1)(b-2)} \\
E(a)=\frac{(b+1)(b-4)(b-5)}{(b-1)(b-2)(b+8)}
\end{gathered}
$$

and

$$
\begin{aligned}
E(b)=\frac{32 b(b-1)(b-2)^{2}}{(b-3)(b-4)^{3}\left\{\left[\frac{d^{2} \ln \Gamma(b-1)}{d b^{2}}\right] 2 b(b-1)-(2 b+1)\right\}}\left[\frac{20\left(3 b^{2}+74 b-40\right)(b-3)^{2}}{(b-4)(b-5)(b-6)(b-7)}\right. \\
\left.\quad-\frac{72(b-3)(b+4)}{(b-4)(b-5)}+\frac{432(b-3)^{2}(b+4)}{(b-4)^{3}(b-5)}-\frac{384(b-3)^{2}(5 b+2)}{(b-4)^{2}(b-5)(b-6)}+\frac{560(b-3)}{(b-4)^{2}}+36\right]^{-1} .
\end{aligned}
$$


The relative efficiency of each parameter is a function of $b$, which itself is a function of the skewness. In table 8 relative efficiencies of each parameter are given for various values of skewness.

From equations 77 through 85 and tables 6 through 8 , it is seen that the relative efficiency of the maximumlikelihood estimate to the moment estimate of each parameter varies inversely with the skewness; that is, the relative efficiency decreases as the skewness increases. The average value of skewness of the low-flow data for the 34 streams was found to be approximately 1. For this value of skewness, the relative efficiencies of the parameters are less than 0.7. The parameters measuring the skewness for the probability distributions have relative efficiencies less than 0.2 . By using 0.5 as the average relative efficiency for all the parameters of a given probability distribution, the method of moments can be considered as using only half of the available information in a given record of size $N$.

\section{APPLICATION OF THE METHOD OF MAXIMUM IIKELIHOOD}

The method of maximum likelihood minimizes the effect of the larger values on the lower limit, since this method gives less weight to the larger values than the method of moments. Lessening the weight of the larger values reduces the estimated skewness. To demonstrate the application of the method of maximum likelihood, the low-flow data for the Kootenai River at Leonia, Idaho, were fitted with a Pearson Type III distribution based on maximum-likelihood estimates of the parameters of the distribution. This set of data was used because it is a good example of the situation where the largest value deviates excessively with the other values.

In order to fit the Pearson Type III distribution to the data by the method of maximum likelihood, it is necessary to solve the following three simultaneous nonlinear equations for the values of $m, a$, and $b$.

$$
\begin{gathered}
\frac{\partial L}{\partial m}=-b \sum_{i=1}^{N}\left(\frac{1}{x_{i}-m}\right)+\frac{N}{a}=0, \\
\frac{\partial L}{\partial a}=-\frac{N}{a}(b+1)+\frac{1}{a^{2}} \sum_{i=1}^{N}\left(x_{i}-m\right)=0, \\
\frac{\partial L}{\partial b}=-N \frac{d \ln \Gamma(b+1)}{d b}+\sum_{i=1}^{N} \ln \left(\frac{x_{i}-m}{a}\right)=0 .
\end{gathered}
$$

The solution of this set of simultaneous equations was obtained by trial and error. The comparison of the maximum likelihood estimates $m, a$, and $b$ with the moment estimates of $m, a$, and $b$ is summarized in table 9.
From table 9, it is seen that the maximum-likelihood estimate of $b$ is larger than the moment estimate of $b$. Since $b$ varies inversely with the skewness, the method of maximum likelihood gives an estimate of the skewness which is less than that given by the method of moments. This reduction of the estimate of the skewness leads to a reduction in the estimate of the lower limit. The minimum observed low flow for the Kootenai River at Leonia, Idaho, was $1,070 \mathrm{cfs}$. By the method of moments, the lower limit was found to be $1,348 \mathrm{cfs}$; whereas, by the method of maximum likelihood, the lower limit was found to be $809 \mathrm{cfs}$.

\section{SUMMARY AND CONCLUSIONS}

\section{PROBABILITY DISTRIBUTION OF LOW FLOWS}

For purposes of estimating low flows having certain probabilities of occurrence and for making tests of significance of such estimates, it is necessary to determine the underlying probability distribution of the data. The low-flow data for 34 streams were found to be nonnormally distributed with an average skewness of approximately 1 . Four theoretical probability distributions were investigated: (a) Gumbel's limited distribution of the smallest value, (b) 3-Parameter LogNormal, (c) Pearson Type III, and (d) Pearson Type V. Each of these distributions is skewed, has a finite lower limit, and is defined by three parameters.

In order to evaluate the applicability of these four theoretical probability distributions to low-flow data, investigations were made relative to two criteria. The first criterion was based on the relation between the observed minimum low flow and the lower limits of the theoretical probability distributions. A theoretical probability distribution was considered to be inapplicable if either the minimum observed low flow was less than the lower limit or if the lower limit was negative. The second criterion was based on the observed relation between skewness and kurtosis with respect to the theoretical relations between skewness and kurtosis. A unique relation between skewness and kurtosis exists for each of the theoretical probability distributions. The theoretical probability distribution whose skewnesskurtosis relation best fitted the observed values of skewness and kurtosis was considered as most representative of the probability distribution of low flows.

On the basis of these two criteria, it was found that the Gumbel and the Pearson Type III distributions were more representative of the probability distribution of low flows than either the 3-Parameter Log-Normal or the Pearson Type $\mathrm{V}$ distributions. Within the range of cumulative probabilities (based on the lengths of low-flow records), the Gumbel and Pearson Type III 
distributions are nearly coincident. Thus the lowflow data can be represented equally well by either distribution.

The lower limits of the four theoretical probability distributions are functions of the skewness and vary directly with the skewness. Hence, for large values of skewness, the lower limits are likely to exceed the observed minimum low flow. In those cases where the lower limits of the Gumbel and the Pearson Type III distributions exceeded the observed minimum low flows, the large values of skewness were attributable to excessive deviations of the larger low flows from the other values. By means of random sampling from a Pearson Type III distribution having a skewness of 1 , it was concluded that such excessive deviations can be attributed to chance.

\section{METHOD OF MAXIMUM LIKELIHOOD}

The above investigations were based on the comparison of parameters estimated by the method of moments. An alternate method of estimating the parameters is that of maximum likelihood. The method of maximum likelihood provides estimates of the parameters such that their variances are a minimum. Also, the method of maximum likelihood provides estimates of the lower limits, which are always less than the minimum observed value.

The increase in information obtained by using the method of maximum likelihood was investigated by comparing the variances of the moment estimates \with the maximum-likelihood estimates of the parameters of the theoretical probability distributions. The ratio of the variance of the maximum-likelihood estimate to the variance of the moment estimate is defined as the relative efficiency between the two methods. This ratio is a fuaction of and varies inversely with the skewness.

The average value of skewness for the low-flow data of 34 streams was found to be approximately 1 . For this value of skewness, the average relative efficiency for the three parameters of each theoretical probability distribution was found to be less than 0.5. Thus the method of moments utilizes less than half of the available information in a set of low-flow data.
Maximum-likelihood estimates of the parameters of the theoretical probability distributions require the solution of three simultaneous nonlinear equations. These solutions are difficult to obtain since they require an iterative process; however, this difficulty can be overcome by the use of high-speed electronic computors. The small relative efficiency of the method of moments with respect to the method of maximum likelihood indicates that future studies dealing with the probability distribution of low flows might best be made by using the method of maximum likelihood. This is particularly true when parameters involving high-order moments are used.

TABLE 1.-Records of annual minimum daily low flows

\begin{tabular}{|c|c|c|c|}
\hline Gaging station & $\begin{array}{c}\text { Period } \\
\text { of } \\
\text { record }\end{array}$ & $\begin{array}{l}\text { Length } \\
\text { of record } \\
\text { (years) }\end{array}$ & $\begin{array}{c}\text { Drainage } \\
\text { area } \\
\text { (sq mi) }\end{array}$ \\
\hline 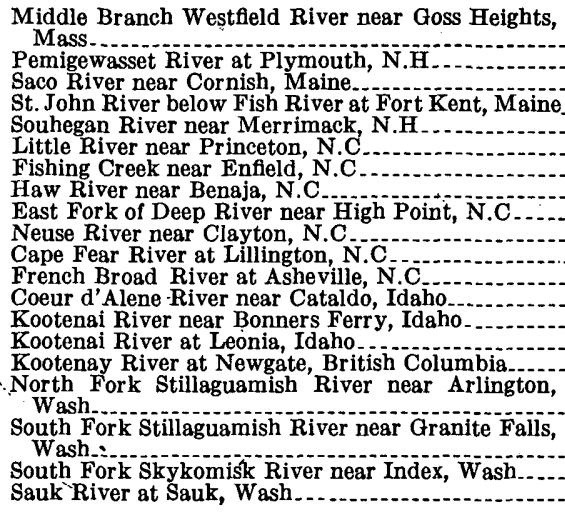 & $\begin{array}{l}1910-55 \\
1903-55 \\
1916-50 \\
1926-50 \\
1909-55 \\
1930-53 \\
1930-53 \\
1928-53 \\
1928-53 \\
1927-52 \\
1927-52 \\
1903-53 \\
1923-50 \\
1928-50 \\
1928-50 \\
1930-50 \\
1928-50 \\
1928-50 \\
1914-50 \\
1930-50\end{array}$ & $\begin{array}{l}45 \\
52 \\
34 \\
24 \\
46 \\
23 \\
23 \\
25 \\
25 \\
25 \\
25 \\
50 \\
27 \\
22 \\
22 \\
20 \\
22 \\
22 \\
36 \\
20\end{array}$ & $\begin{array}{c}52.6 \\
522 \\
1,298 \\
5,690 \\
171 \\
229 \\
521 \\
168 \\
14.7 \\
1,140 \\
3,440 \\
950 \\
1,200 \\
13,000 \\
11,740 \\
7,660 \\
269 \\
199 \\
355 \\
714\end{array}$ \\
\hline
\end{tabular}

TABLE 2.-Records of annual minimum 7-day low flows

\begin{tabular}{|c|c|c|c|}
\hline Gaging stations & $\begin{array}{l}\text { Period } \\
\text { of } \\
\text { record }\end{array}$ & $\begin{array}{l}\text { Length } \\
\text { of record } \\
\text { (years) }\end{array}$ & $\begin{array}{c}\text { Draingge } \\
\text { area } \\
\text { (sq mi) }\end{array}$ \\
\hline 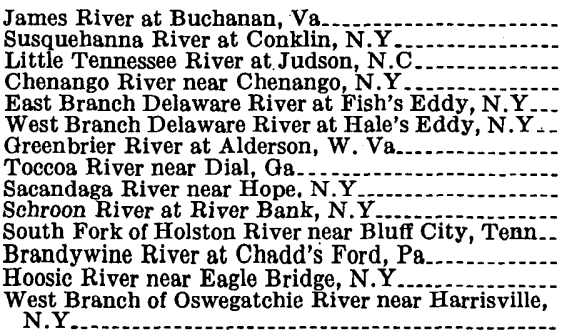 & $\begin{array}{l}1898-50 \\
1919-52 \\
1896-41 \\
1913-52 \\
1913-52 \\
1913-52 \\
1907-54 \\
1913-55 \\
1912-54 \\
1908-53 \\
1901-49 \\
1911-54 \\
1911-53 \\
1916-53\end{array}$ & $\begin{array}{l}52 \\
40 \\
46 \\
40 \\
40 \\
40 \\
48 \\
43 \\
43 \\
46 \\
49 \\
44 \\
43 \\
38\end{array}$ & $\begin{array}{r}2,084 \\
2,240 \\
664 \\
1,492 \\
783 \\
593 \\
1,357 \\
177 \\
491 \\
527 \\
813 \\
287 \\
510 \\
258\end{array}$ \\
\hline
\end{tabular}


TABLE 3.-Coefficients of skewness of low-flow data with 90-percent, 95-percent, and 99-percent confidence limits

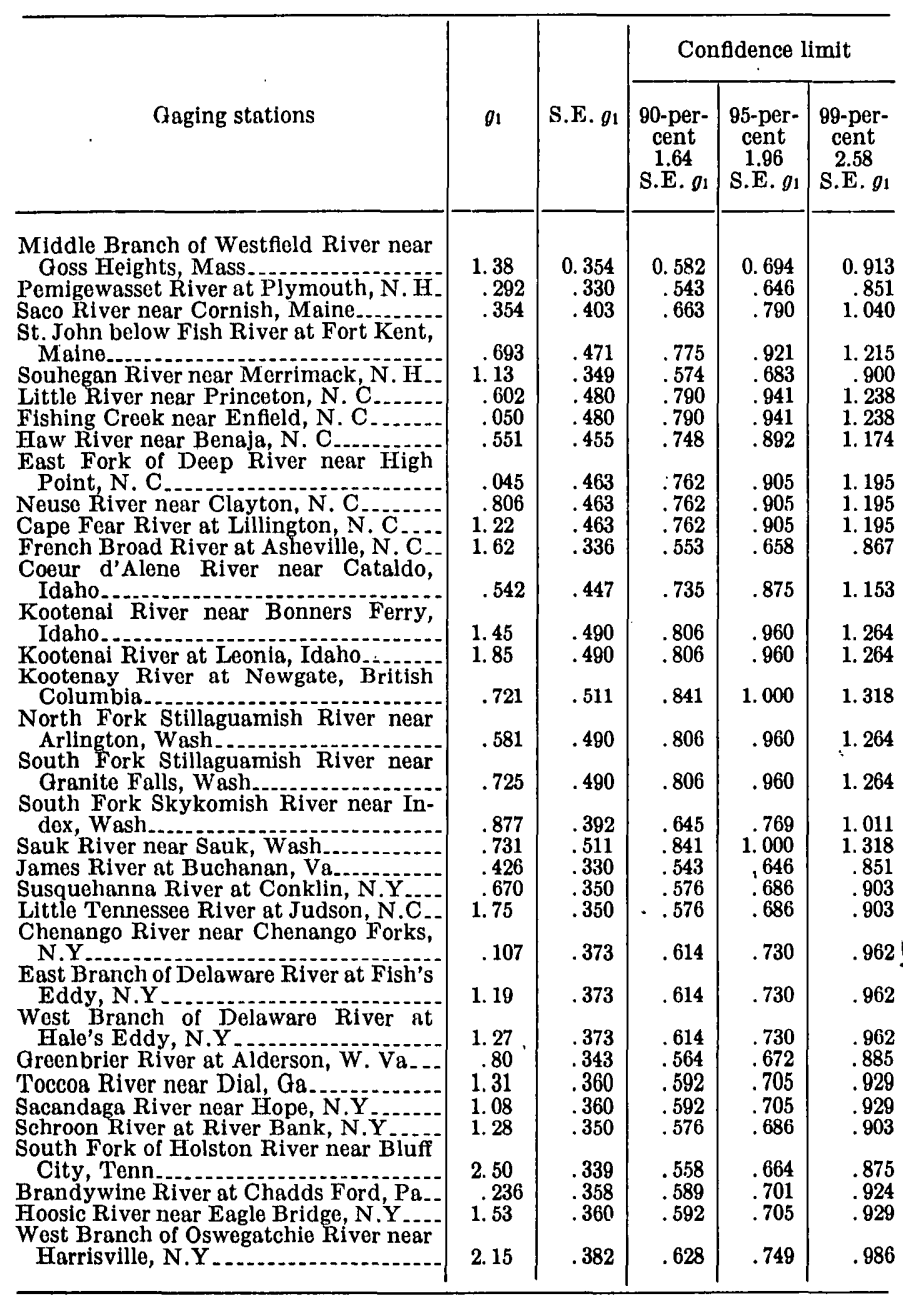

TABLE 4.-Coefficients of kurtosis of low-flow data with 90-percent, 95-percent, and 99-percent confidence limits

\begin{tabular}{|c|c|c|c|c|c|}
\hline \multirow[b]{2}{*}{ Gaging stations } & \multirow[b]{2}{*}{$g_{2}$} & \multirow[b]{2}{*}{ S.E.g2 } & \multicolumn{3}{|c|}{ Confldence limit } \\
\hline & & & $\begin{array}{c}90 \text {-per- } \\
\text { cent } \\
1.64 \\
\text { S.E. } 62\end{array}$ & $\begin{array}{c}95 \text {-per- } \\
\text { cent } \\
1.96 \\
\text { s.E. } \\
\text {. }\end{array}$ & $\begin{array}{c}\text { 99-per- } \\
\text { cent } \\
2.58 \\
\text { 8.E.02 }\end{array}$ \\
\hline Middle Branch of Westfield River & 154 & 0.693 & 140 & 1358 & \\
\hline Pemigewasset River at Plymouth, & -.11 & $\begin{array}{r}0.045 \\
.649\end{array}$ & $\begin{array}{l}1.140 \\
1.068\end{array}$ & $\begin{array}{l}1.358 \\
1.272\end{array}$ & $\begin{array}{l}1.788 \\
1.674\end{array}$ \\
\hline $\begin{array}{l}\text { Saco River near Cornish, Maine } \\
\text { St. John below Fish River at Fort K }\end{array}$ & -.08 & .788 & 1. 293 & 1.541 & 2.028 \\
\hline $\begin{array}{l}\text { Maine. } \\
\text { Souhegan River near Merrimack }\end{array}$ & .90 & .909 & & 1.782 & 2.345 \\
\hline $\begin{array}{l}\text { Sounegan River near Me } \\
\text { Little River near Princet }\end{array}$ & $\begin{array}{l}83 \\
20\end{array}$ & 987 & $\begin{array}{l}1.130 \\
1.523\end{array}$ & $\begin{array}{l}1.347 \\
1.815\end{array}$ & $\begin{array}{l}1.772 \\
2.389\end{array}$ \\
\hline Fishing Creek near Enfleld,' N.C. & -1.04 & .926 & $\begin{array}{l}1.020 \\
1.523\end{array}$ & $\begin{array}{l}1.810 \\
1.815\end{array}$ & 2.389 \\
\hline Haw River near Benaja, N.C.... & & .894 & 1.471 & 1.752 & 2.307 \\
\hline $\begin{array}{l}\text { East Fork of Deep } 1 \\
\text { Point N C }\end{array}$ & _- & & & & \\
\hline Neuse River near Clayton, N.C. & $\begin{array}{l}-.58 \\
-.52\end{array}$ & .894 & $\begin{array}{l}1.471 \\
1.471\end{array}$ & $\begin{array}{l}1.752 \\
1.752\end{array}$ & 2.307 \\
\hline Cape Fear River at Lillington, N. & .02 & .894 & $\begin{array}{l}1.471 \\
1.471\end{array}$ & 1. 1552 & 2.307 \\
\hline French Broad River at Asheville, & .06 & .660 & $\begin{array}{l}1.086 \\
1.086\end{array}$ & 1. 294 & 1.703 \\
\hline Coeur d'Alene River near Cataldo, & & & & & \\
\hline Kootenai River near Bon & .71 & .865 & 1.423 & 1.695 . & 2. \\
\hline Idaho & 3. 55 & .042 & 1.550 & 1.846 & 2.4 \\
\hline Kootenai River at Leonia, Idaho. & 6.40 & .842 & 1.150 & 1.846 & 2.430 \\
\hline $\begin{array}{l}\text { Kootenay River at Newgate, } B \\
\text { Columbia }\end{array}$ & 1.3 & .980 & 1.61 & 1. $\theta$ & \\
\hline North Fork Stillaguamish River & & .800 & 1.012 & 1. $\forall$ & \\
\hline Arlington, Wash & .02 & .942 & 1.550 & 1.846 & 2.430 \\
\hline $\begin{array}{l}\text { South Fork Stillaguamish River n } \\
\text { Granite Falls, Wash...................... }\end{array}$ & .5 & .942 & 1. 550 & 1.846 & 2.4 \\
\hline South Fork Skykomish River n & & & & & \\
\hline Index, Wash... & 33 & .766 & 1. 260 & 1.501 & 1.976 \\
\hline Sauk River near & & .980 & 1.612 & 1. 921 & 2. 528 \\
\hline ames River at & 64 & .645 & 1.061 & 1. 264 & 1. 664 \\
\hline hanna River at Conklin, N. & .27 & .687 & 1.130 & 1.347 & 1.772 \\
\hline Little Tennessee River at Judson, & -2.33 & .687 & 1.130 & 1.347 & 1.772 \\
\hline Chenango River near & & 720 & & & 18 \\
\hline East Branch of Delaware River at $\mathrm{F}$ & & 730 & 1.201 & 1.4 & 1.883 \\
\hline Eddy, N.Y & .73 & .730 & 1.201 & 1.431 & 1. 88.3 \\
\hline West Branch of Delaware River at & & & & & \\
\hline Greenbrier River at Alderson, w. & $\begin{aligned} 1.04 \\
-.11\end{aligned}$ & .730 & $\begin{array}{l}1.201 \\
1.107\end{array}$ & $\begin{array}{l}1.431 \\
1.319\end{array}$ & $\begin{array}{l}1.883 \\
1.736\end{array}$ \\
\hline Toccoa River near Dial, Ga. & 1.50 & .707 & 1.163 & 1.386 & 1.824 \\
\hline Sacandaga River near Hope, N.Y & .93 & .707 & 1.163 & 1.386 & 1.824 \\
\hline $\begin{array}{l}\text { Schroon River at River Bank, N.Y } \\
\text { South Fork of Holston River near B }\end{array}$ & -2.14 & .687 & 1.130 & 1.347 & 1.772 \\
\hline City, Tenn & 9.65 & .668 & 1.090 & 1.309 & \\
\hline Brandywine Riv & -.49 & .700 & 1.152 & 1.372 & 1.806 \\
\hline Hoos & 2. 29 & .707 & 1. 163 & 1.386 & 1.824 \\
\hline $\begin{array}{l}\text { West Branch of Oswegatchie River near } \\
\text { Harrisville, N.Y. }\end{array}$ & 7.09 & .748 & 1.230 & 1.466 & 950 \\
\hline & & & & & \\
\hline
\end{tabular}


TABLE 5-Observed and theoretical standardized low limits

\begin{tabular}{|c|c|c|c|c|c|c|}
\hline Gaging stations & $-t_{0}$ & $-t_{G}$ & $-t_{I I I}$ & $-t_{L N}$ & $-t_{v}$ & $-t^{\prime}$ \\
\hline $\begin{array}{l}\text { Middle Branch of Westfield River near } \\
\text { Goss Heights, Mass }\end{array}$ & 1.33 & 11.3 & 11.2 & 2.31 & 4.20 & 1.33 \\
\hline PemigewassetRiveratPlymouth, N.H.- & 2.36 & 2.5 & 2.6 & 10.5 & 13.5 & 3.35 \\
\hline $\begin{array}{l}\text { Saco River near Cornish, Maine } \\
\text { St. John below Fish River at Fort Kent, }\end{array}$ & 1.74 & 2.4 & 2.4 & 8.6 & 11.5 & 3.46 \\
\hline Maine & 1.75 & 1.85 & 11.7 & 4.4 & 6.40 & 3.14 \\
\hline ear Merrimack, I & 1. 26 & 1.42 & & 2.78 & 4.50 & 3.08 \\
\hline $\begin{array}{l}\text { Little Rive } \\
\text { Fishing Cre }\end{array}$ & 1.58 & 2.00 & 1.8 & 5.00 & 7. 20 & 1.66 \\
\hline $\begin{array}{l}\text { Fishing Creek near Enfield, N.C.- } \\
\text { Haw River near Benaja, N.C....-. }\end{array}$ & 1.58 & 3.1 & 6.3 & 60 & 81 & 1.88 \\
\hline East Fork of Deep River near High & & 2.00 & & & 7.80 & 2.08 \\
\hline Point, N.C.- & 1.99 & 3.15 & 6.6 & .67 & & 3.79 \\
\hline Neuse River n & 1.34 & 1.70 & 1.55 & 3. 75 & 5.7 & 1. 90 \\
\hline $\begin{array}{l}\text { at Lillington, } \mathrm{N} \\
\text { iver at Asheville. }\end{array}$ & 1.23 & 1.35 & 1.28 & 2.69 & 4.3 & 1.59 \\
\hline Coeur d'Alene River near Catald & 1.60 & 11.15 & 11.11 & 2.0 & 3.75 & 2.02 \\
\hline $\begin{array}{l}\text { Idaho } \\
\text { Kootenai River near Bon }\end{array}$ & 1.89 & 2.05 & 1.9 & 5.60 & 7.8 & 3.97 \\
\hline $\begin{array}{l}\text { Kootenai River near Bonners } \\
\text { Idaho. }\end{array}$ & 1. & & & & & \\
\hline i River at Leon & 1.45 & i 1.03 & 11.05 & 1.80 & 3. 5 & $\begin{array}{l}3.10 \\
2.88\end{array}$ \\
\hline Koote & & & & & & \\
\hline $\begin{array}{l}\text { Columbia } \\
\text { North Fork Stillagu }\end{array}$ & 1.78 & 1.82 & 11.65 & 4. 20 & 6.2 & 4.49 \\
\hline Arlington, Wash & 1.66 & 2. 00 & 1.82 & 5. 20 & 7.4 & 3.93 \\
\hline $\begin{array}{l}\text { South Fork Stillaguamish River } \\
\text { Granite Falls, Wash..................... }\end{array}$ & & & 1.65 & 4.20 & 0.2 & 3.02 \\
\hline $\begin{array}{l}\text { South Fork Skyomish River near Inde } \\
\text { Wash }\end{array}$ & & 1.6 & & & & 0.02 \\
\hline $\begin{array}{l}\text { W } \\
\text { Sauk }\end{array}$ & 1.62 & 1.65 & 11.5 & 3.50 & 5. 3 & 4.24 \\
\hline $\begin{array}{l}\text { Sauk } \\
\text { Jame }\end{array}$ & 1.72 & 1.80 & $\begin{array}{rl}1 & 1.63 \\
2.15\end{array}$ & $\begin{array}{l}4.15 \\
7.20\end{array}$ & $\begin{array}{l}6.1 \\
9.8\end{array}$ & $\begin{array}{l}4.53 \\
4.15\end{array}$ \\
\hline nklin, $\mathrm{N}$ & 1. 59 & 1.88 & 1.7 & 4.45 & 6. & 2.46 \\
\hline dson, $\mathrm{N}$ & 1.70 & $\begin{array}{lll}1 & 1.10\end{array}$ & 11.08 & 1. 90 & 3.6 & 2.98 \\
\hline $\begin{array}{l}\text { Chenango River near Chenango Forks, } \\
\text { N.Y }\end{array}$ & & & & 28 & 38 & 2.97 \\
\hline of Delaware River a & & & & & & \\
\hline $\begin{array}{l}\text { Fish's Eddy, N.Y. } \\
\text { West Branch of Del }\end{array}$ & 1.11 & 1.38 & 1.3 & 2.65 & 4.4 & 1.90 \\
\hline west Hale's Eddy, N.Y & 1.07 & 1.95 & 1. & 2. & 4 & 1. \\
\hline Greenbrier River at Alderson, W.V & 1.52 & 1.74 & & 3. & 7. & 1.77 \\
\hline & 1.70 & 11.30 & 11.23 & 2. & 4. & 2.97 \\
\hline Sace & 1.45 & 1.46 & 11.35 & 2. & 4. 64 & 2.00 \\
\hline Schroon River & 1.40 & ${ }^{1} 1.32$ & 11.25 & 2.50 & 4. & 2.58 \\
\hline & 1.09 & 1.86 & & & 0 & \\
\hline Bran & 1.71 & 1. & 2. & 12. & 17 & 2. \\
\hline Hoc & 1.33 & 11.17 & 11.15 & 2.15 & 3.9 & 2.92 \\
\hline $\begin{array}{l}\text { West Branch of Oswegatchie River near } \\
\text { Harrisville, N.Y }\end{array}$ & 1.12 & 1.95 & 10.96 & 1.58 & 3.35 & 20 \\
\hline
\end{tabular}

1 Theoretical lower limit is greater than observed minimum low flow.
TABLE 6.-Relative efficiency of moment estimates with respect to maximum-likelihood estimates-Pearson Type III distribution

\begin{tabular}{|c|c|c|c|c|}
\hline$G_{1}$ & $p$ & $E(m)$ & $E(a)$ & $E(p)$ \\
\hline 1 & $\begin{array}{c}0 \\
\stackrel{3}{3} \\
43.44\end{array}$ & $\begin{array}{r}0.000 \\
.500 \\
.954\end{array}$ & $\begin{array}{r}0.250 \\
.571 \\
.937\end{array}$ & $\begin{array}{r}0.000 \\
.154 \\
.821\end{array}$ \\
\hline
\end{tabular}

TABLE 7.-Relative efficiency of moment estimates with respect to maximum-likelihood estimates-3-Parameter Log-Normal distribution

\begin{tabular}{|c|c|c|c|c|}
\hline$G_{1}$ & $\gamma$ & $E(a)$ & $E(m)$ & $E(\sigma)$ \\
\hline & $\begin{array}{r}0.6 \\
.3\end{array}$ & $\begin{array}{r}0.259 \\
.683\end{array}$ & $\begin{array}{r}0.280 \\
.624\end{array}$ & $\begin{array}{l}0.003 \\
.006\end{array}$ \\
\hline 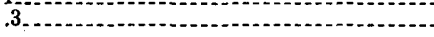 & .1 & .958 & .936 & .874 \\
\hline
\end{tabular}

TABLE 8.-Relative efficiency of moment estimates with respect to maximum-likelihood estimates-Pearson Type $V$ distribution

\begin{tabular}{|c|c|c|c|c|}
\hline$a_{1}$ & $b$ & $E(m)$ & $E(a)$ & $E(b)$ \\
\hline 2-. & $\stackrel{9}{9}$ & $\begin{array}{l}0.371 \\
672\end{array}$ & $\begin{array}{l}0.210 \\
542\end{array}$ & $\begin{array}{l}0.002 \\
.005\end{array}$ \\
\hline 13 & $\begin{array}{r}21 \\
183\end{array}$ & & $\begin{array}{l}.542 \\
.982\end{array}$ & \\
\hline
\end{tabular}

TABLE 9.-Comparison of parameters computed by the methods of maximum-likelihood and moments

\begin{tabular}{|c|c|c|c|c|c|c|c|c|}
\hline & \multicolumn{4}{|c|}{ Pearson Type III } & \multicolumn{4}{|c|}{ 3-Parameter Log-Normal } \\
\hline & $m$ & $a$ & $p$ & $L$ & $m$ & $a$ & $\sigma$ & $L_{L N}$ \\
\hline likelihood.. & $\begin{array}{r}809 \\
1348\end{array}$ & $\begin{array}{l}360 \\
695\end{array}$ & $\begin{array}{r}2.755 \\
.169\end{array}$ & $\begin{array}{l}-141.28 \\
-172.30\end{array}$ & $\begin{array}{l}7.38 \\
7.09\end{array}$ & $\begin{array}{l}435 \\
794\end{array}$ & $\begin{array}{r}0.395 \\
.514\end{array}$ & $\begin{array}{l}-173.02 \\
-173.5\end{array}$ \\
\hline
\end{tabular}




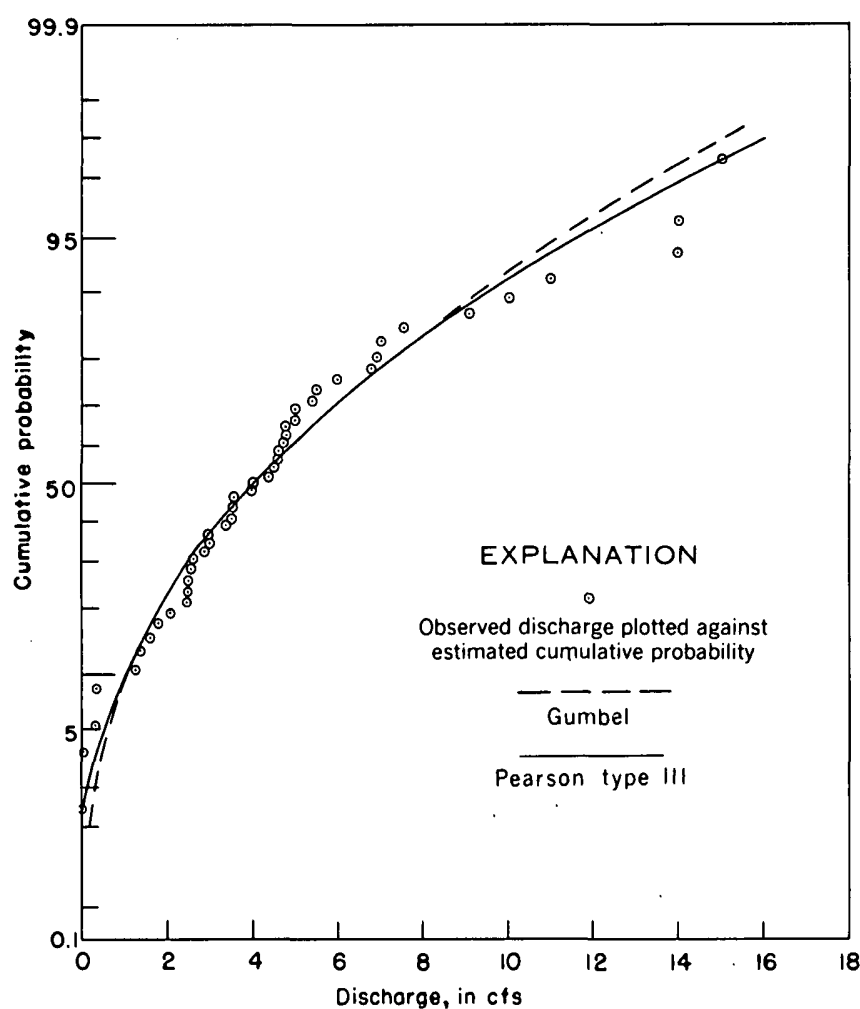

Fiaure 1.-Middle Branch Westfeld River at Goss Heights, Mass.

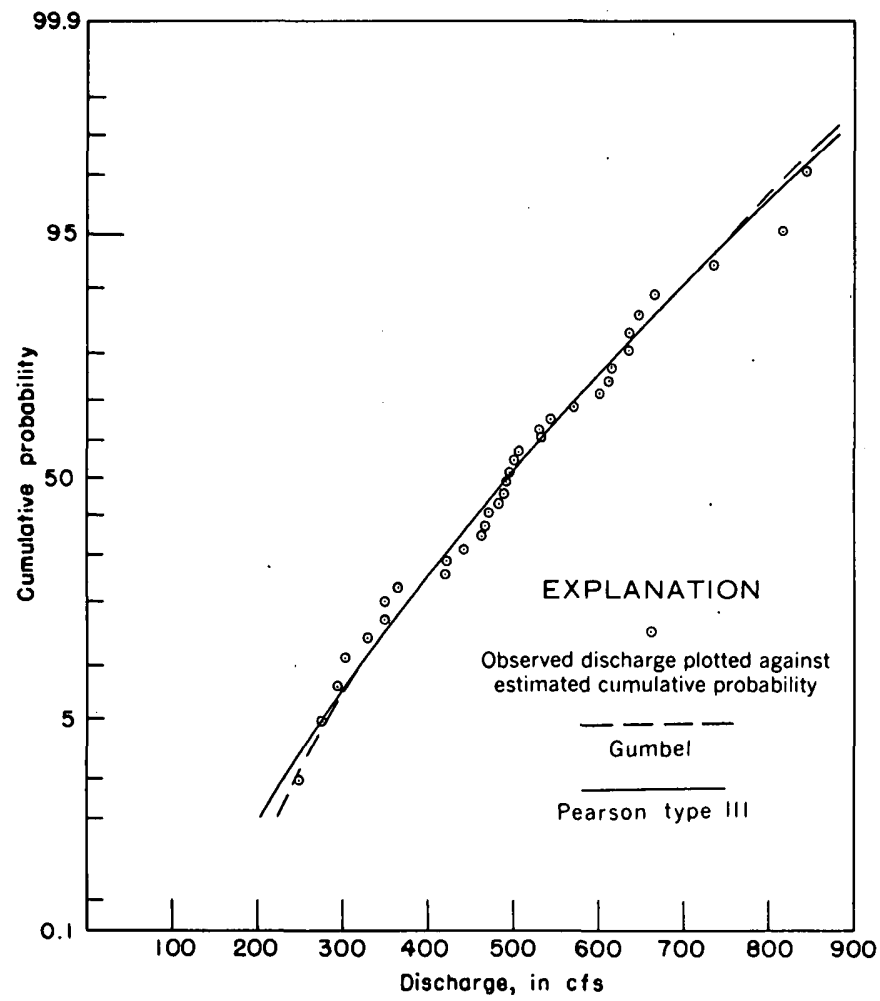

Fraure 3.-Saco River near Cornish, Maine.

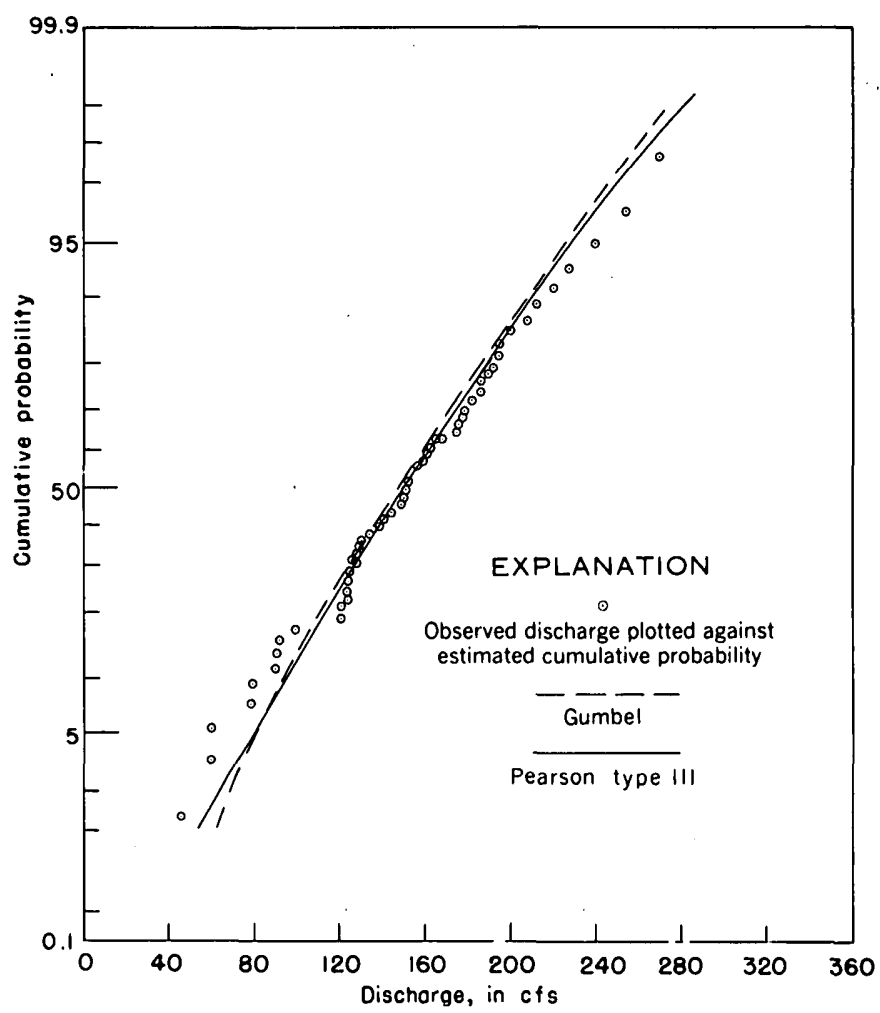

Figure 2:-Pemigewasset River at Plymouth, N.H.

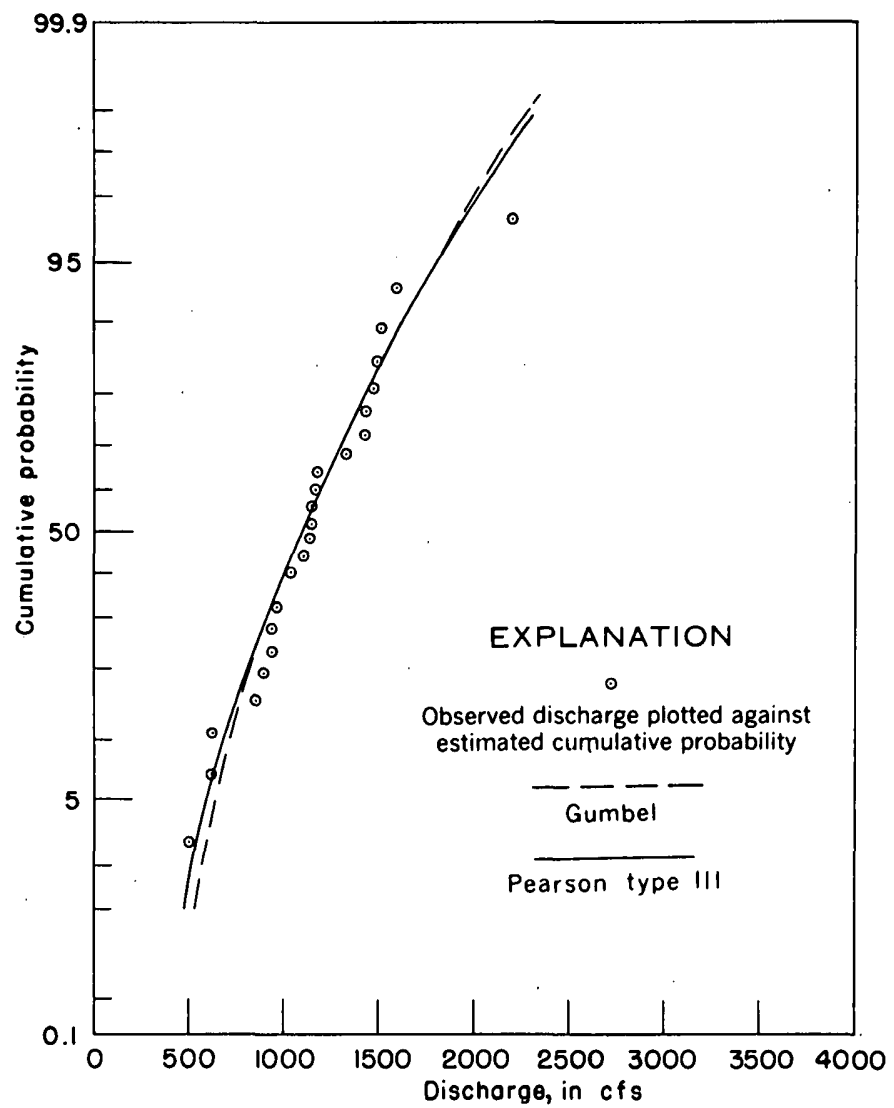

Flaure 4.-St. John River below Fish River at Fort Kent, Maine. ।

LOW-FLOW PROBABILITY DISTRIBUTION 


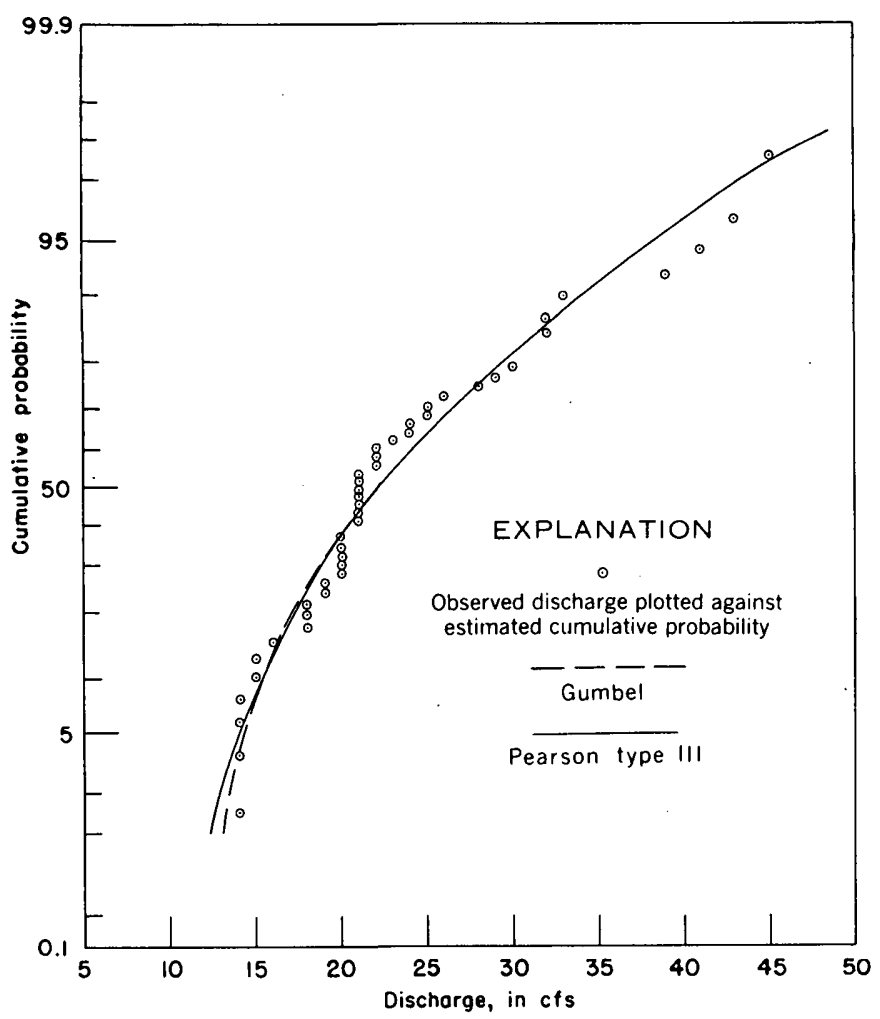

FIGURE 5.-Souhegan River near Merrimack, N.H.

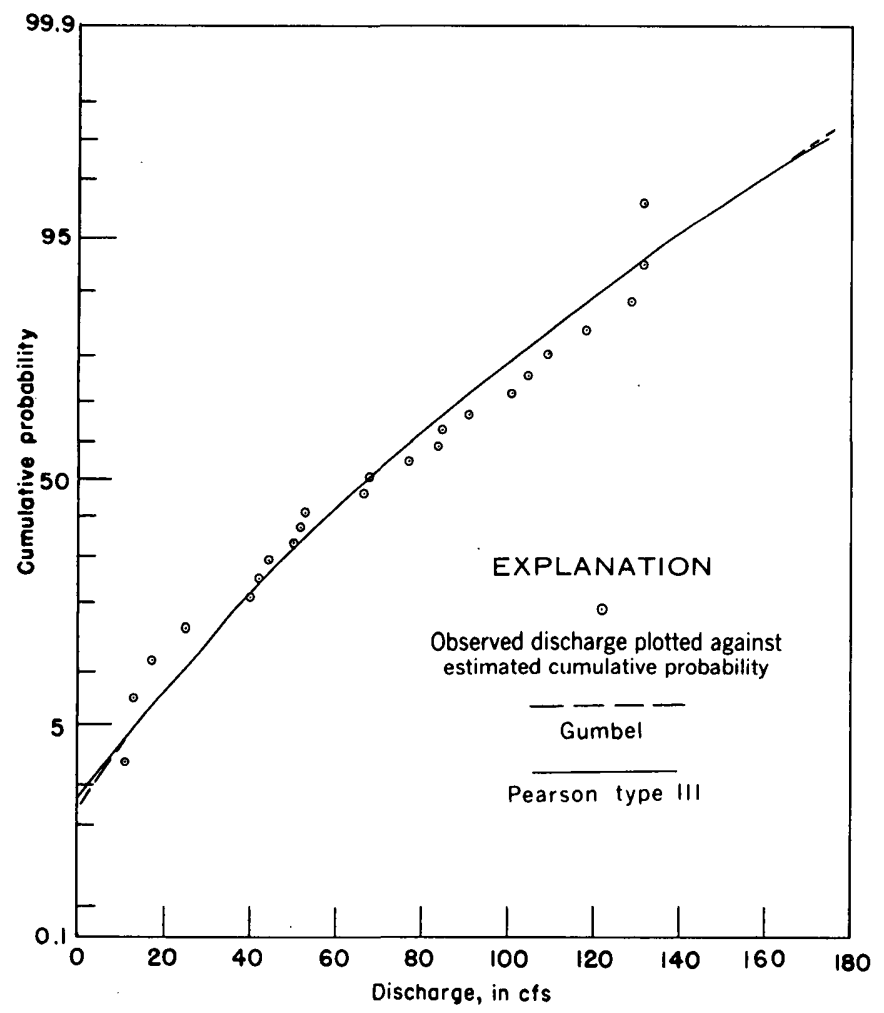

Figure 7.-Fishing Creek near Enfeld, N.C.

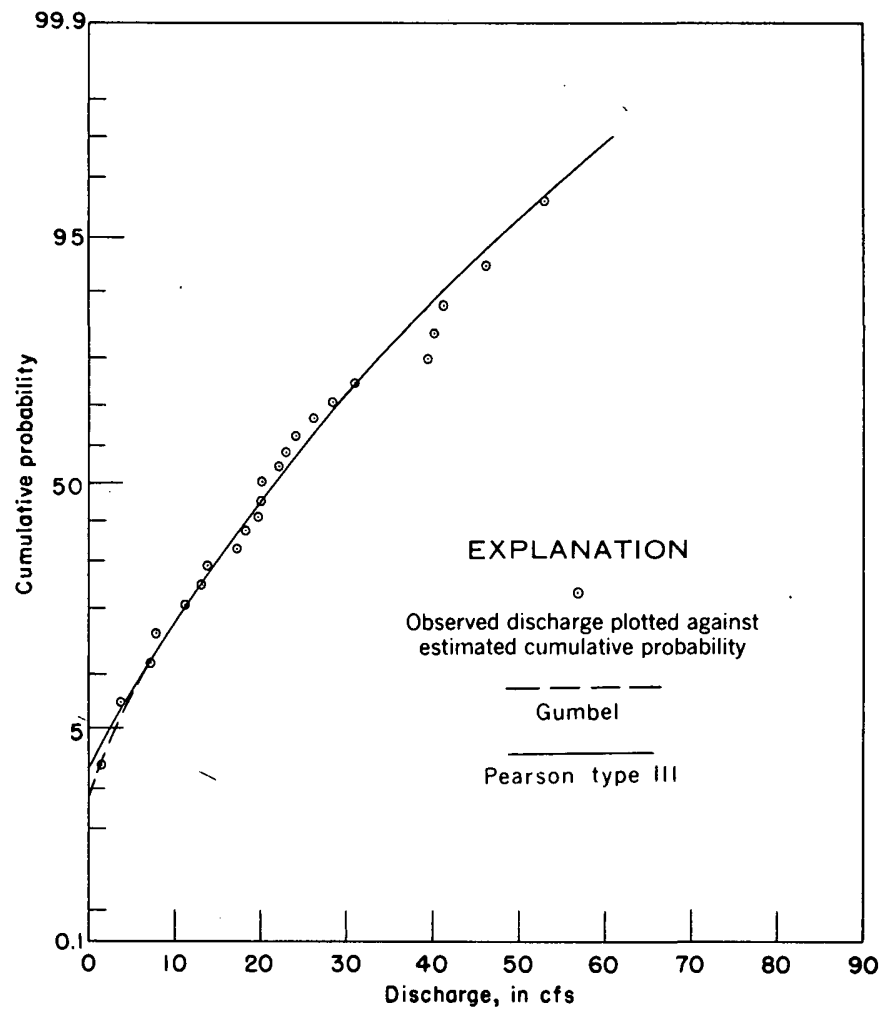

Frgure 6.-Little River near Princeton, N.C.

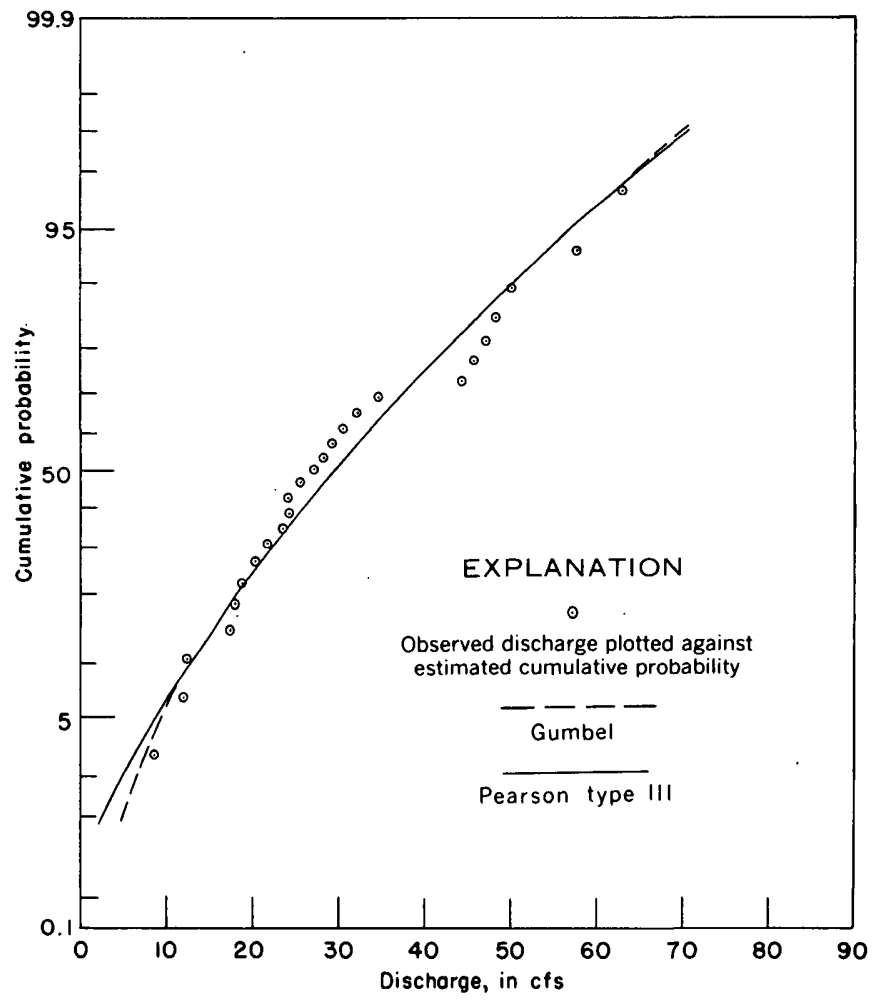

FIGURe 8.-Haw River near Benaja, N.C.

LOW-FLOW PROBABILITY DISTRIBUTION 


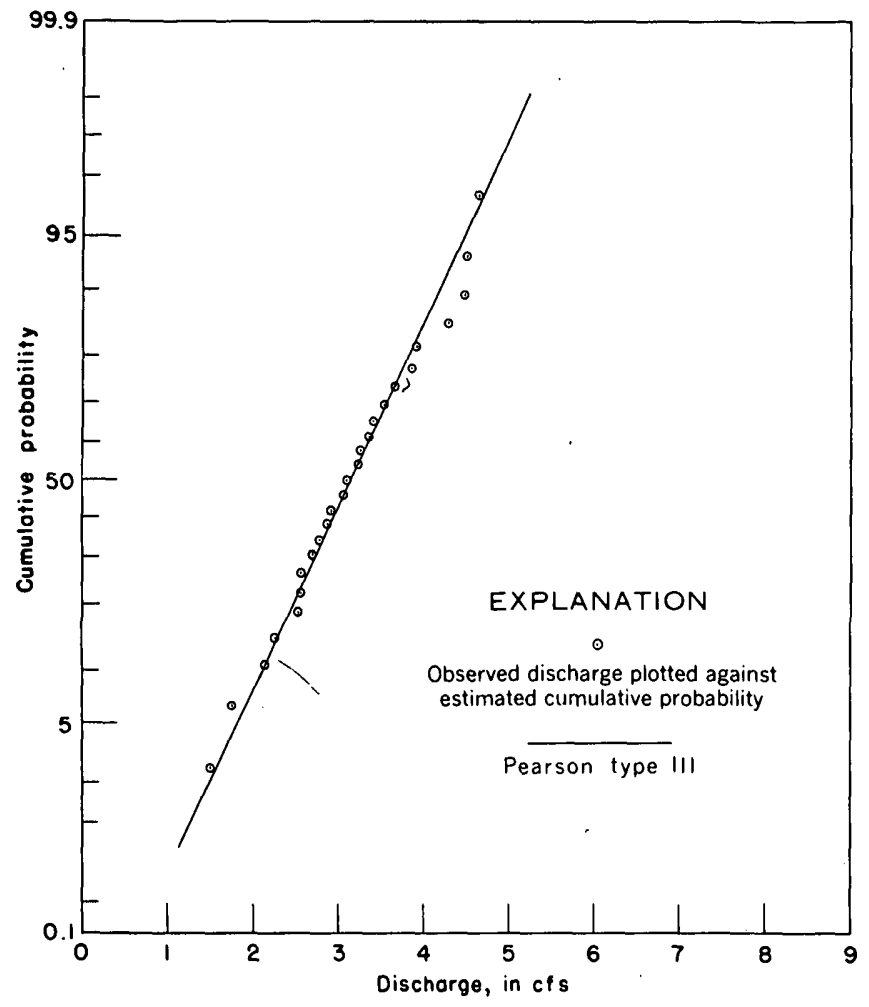

Figure 9.-East Fork of Deep River near High Point, N.C.

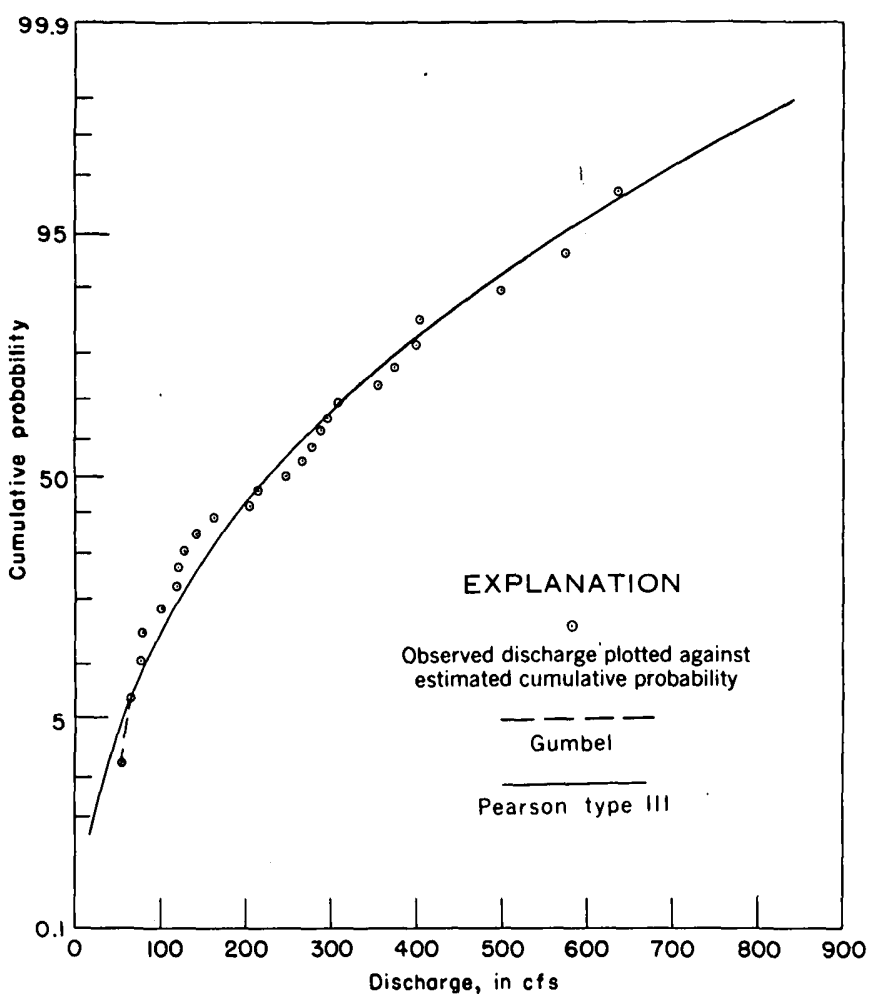

Figure 11.-Cape Fear River at Lillington, N.C.

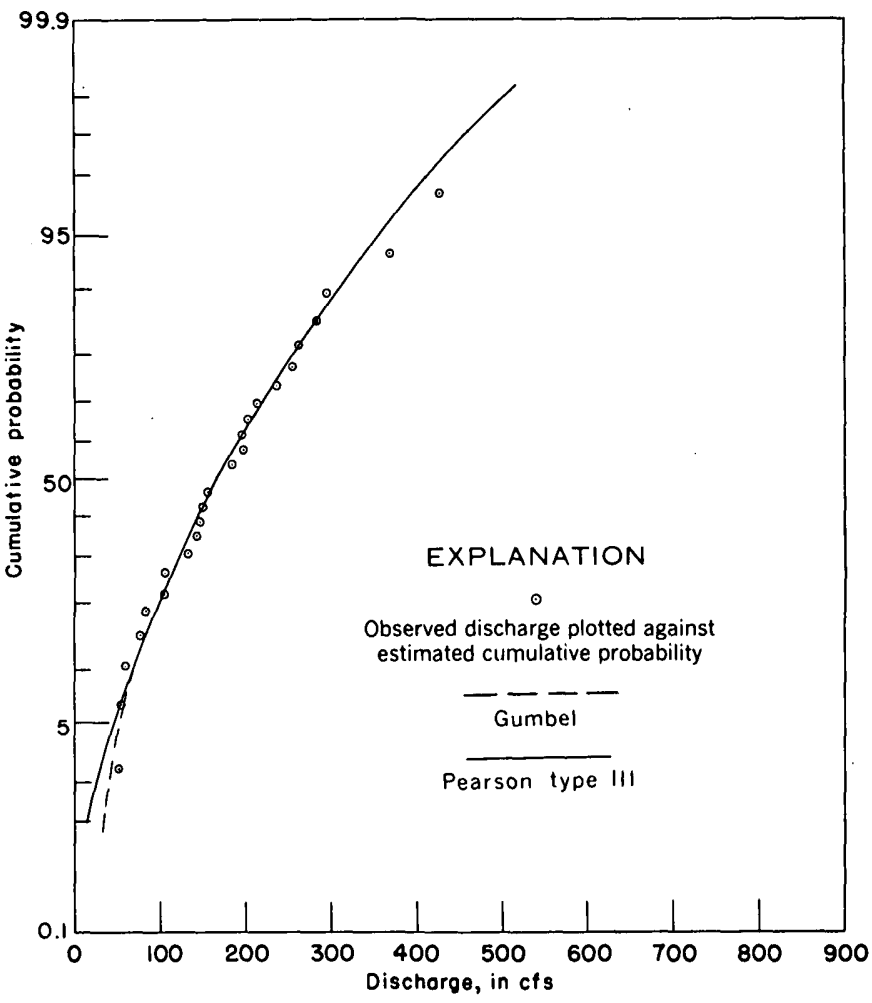

Froure 10.-Neuse River near Clayton, N.C.

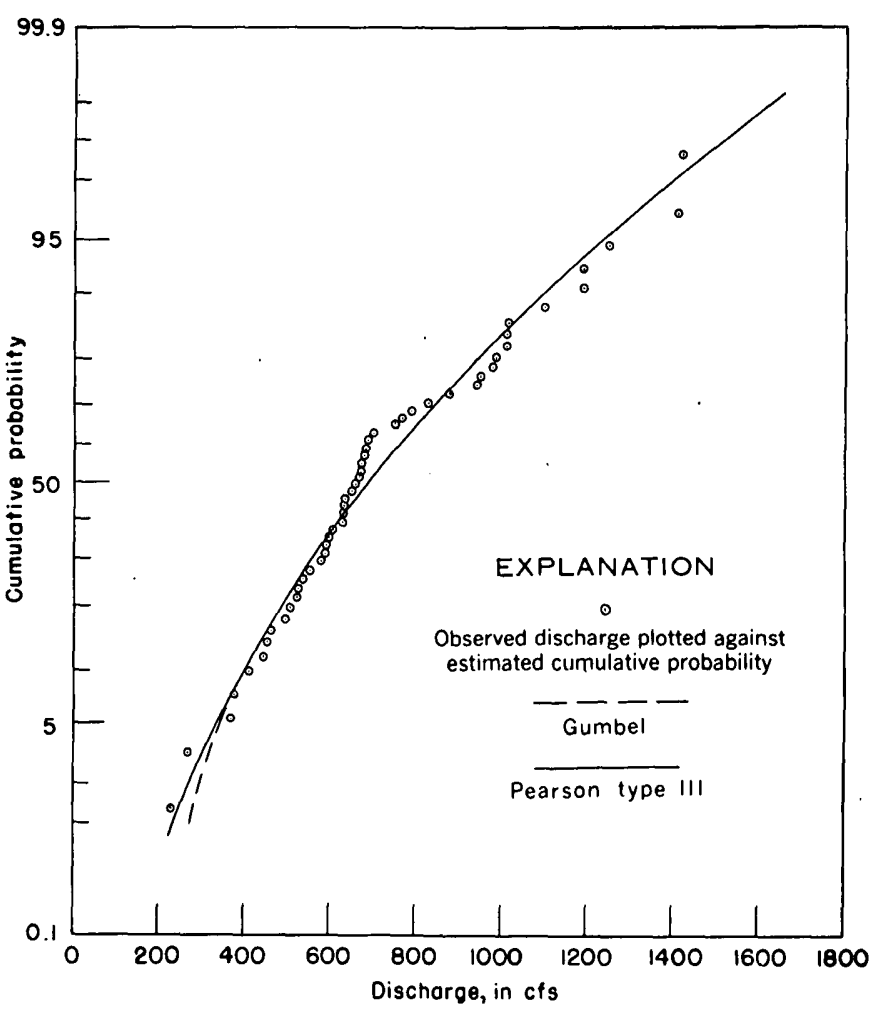

Fioure 12,-French Broad River near Asheville, N.C. 


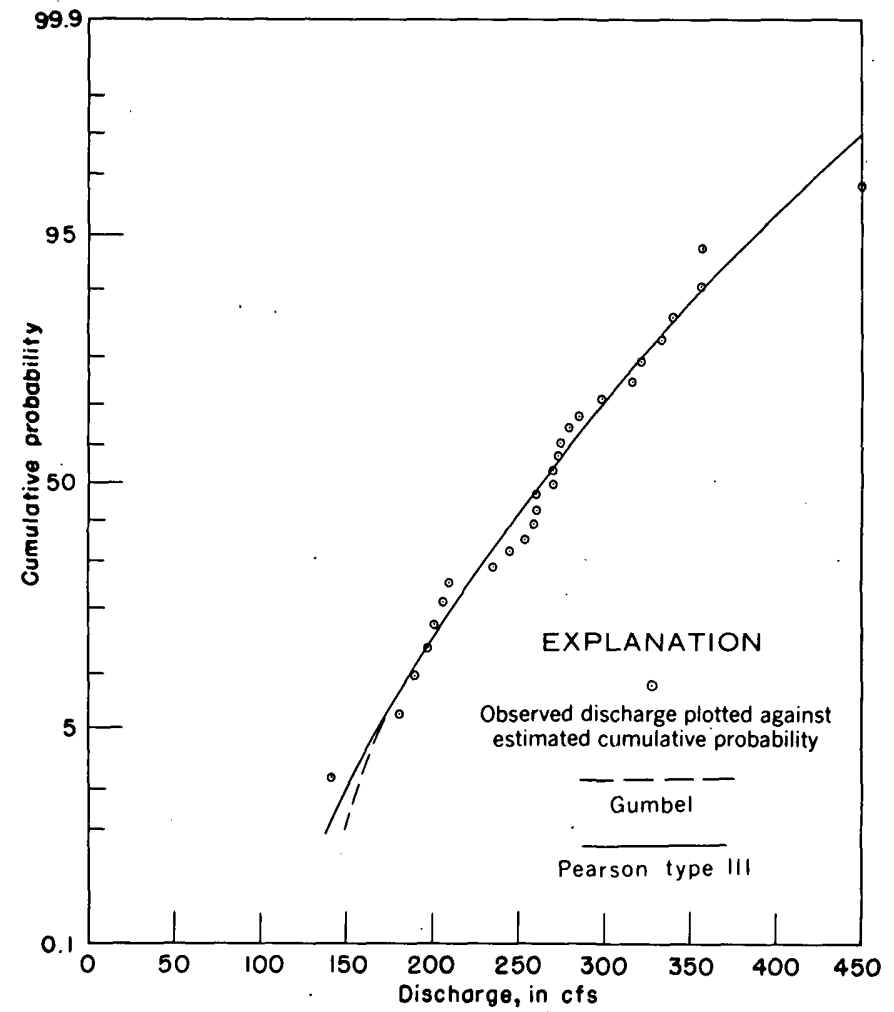

Fiadre 13.-Coeur d'Alene River near Cataldo, Idaho.

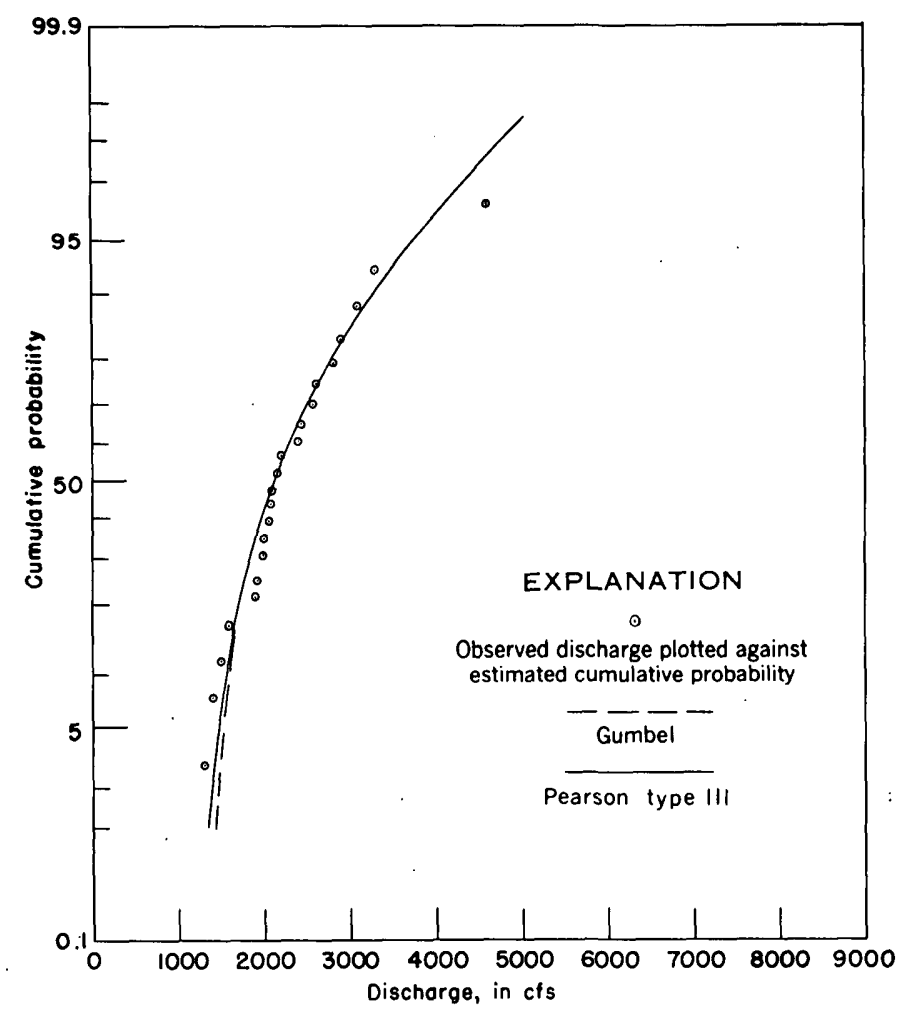

FIGORE 15.-Kootenai River at Bonner's Ferry, Idaho.

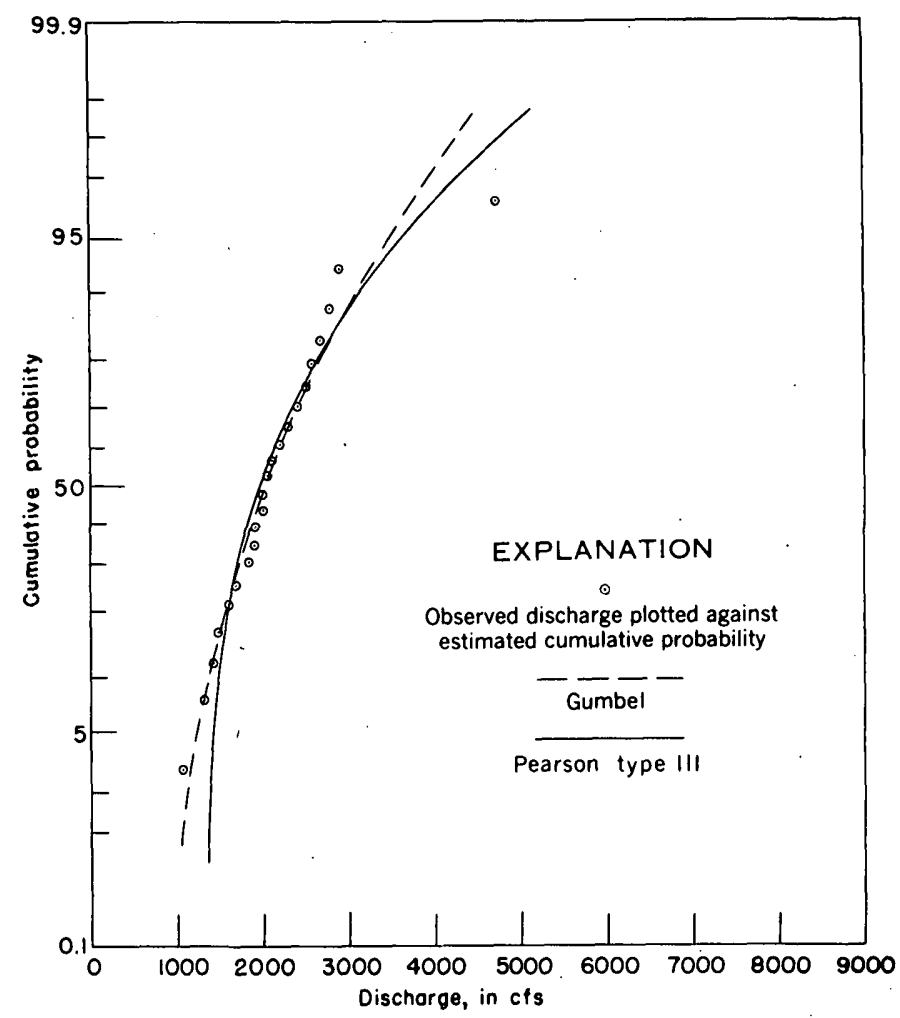

FroURE 14.-Kootenai River near Leonia, Idaho.

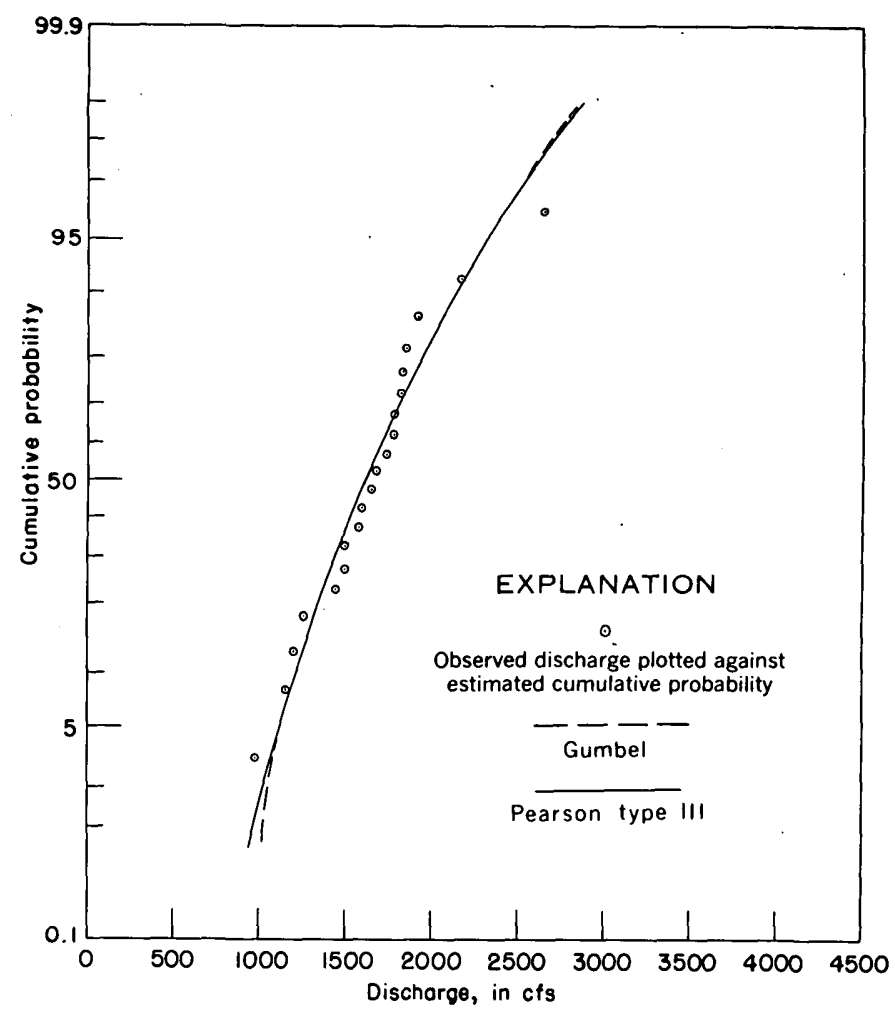

Figure 16.-Kootenay River near Newgate, British Columbia. 


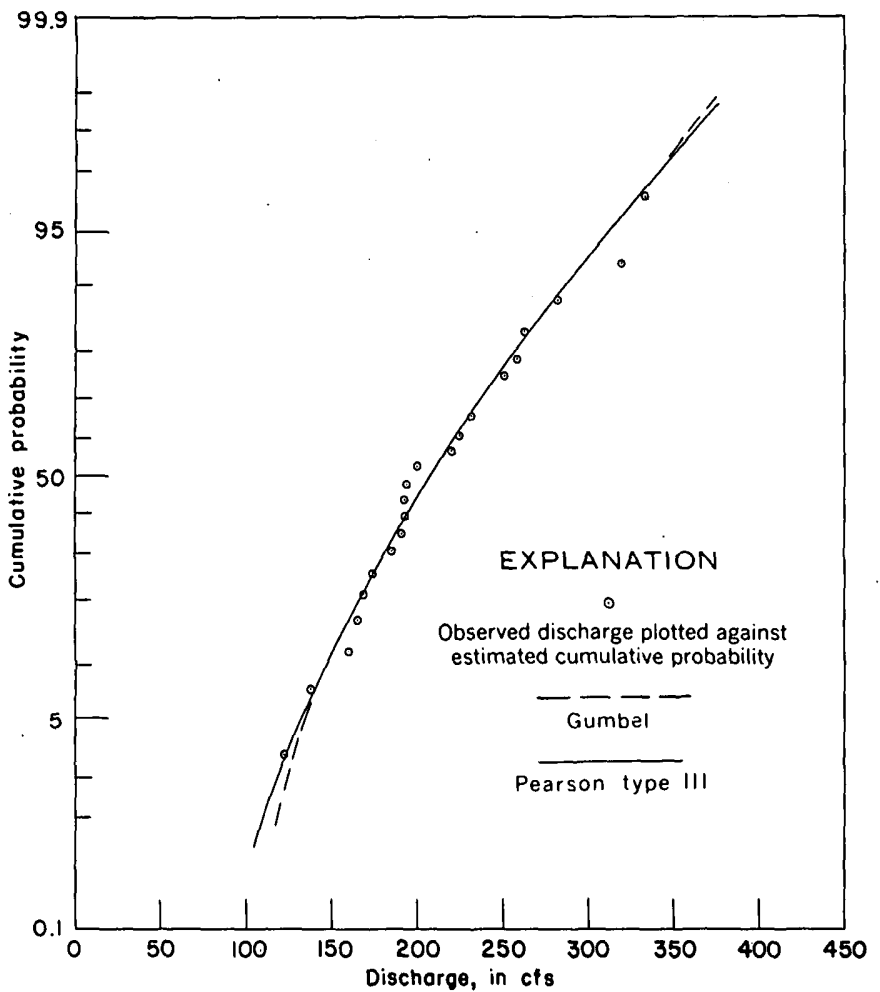

FiquRE 17.-North Fork Stillaguamish River near Arlington, Wash.

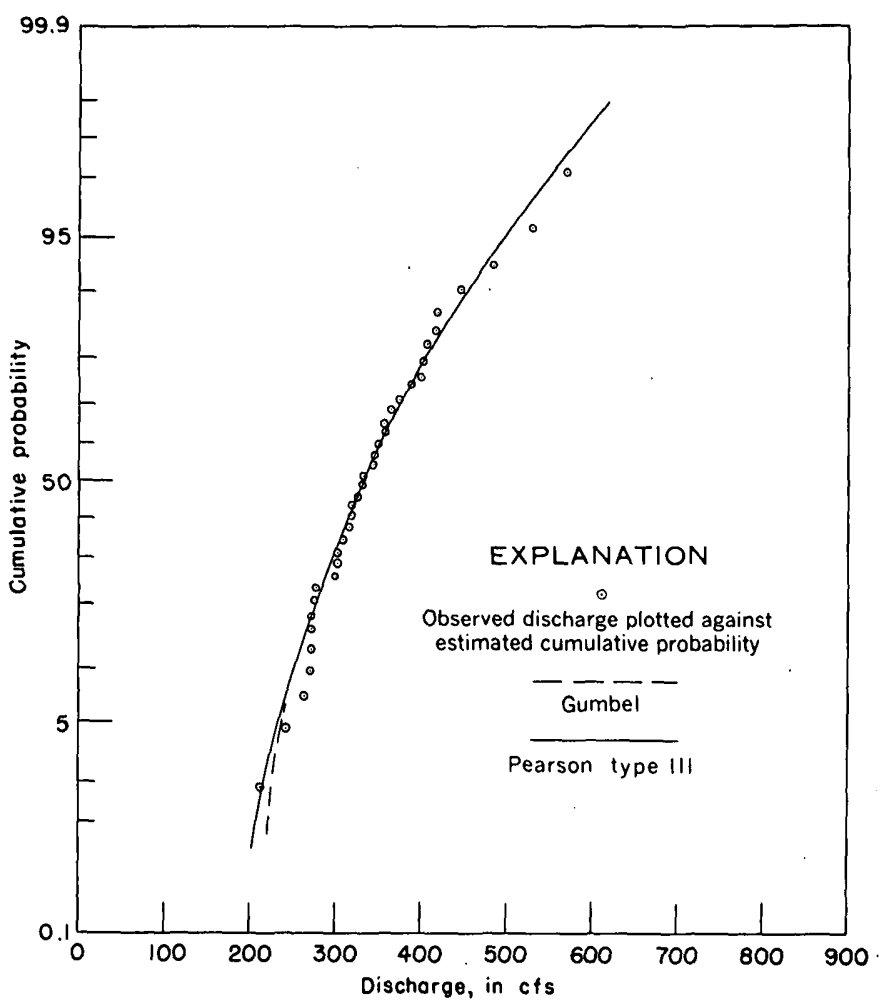

Figure 19,-South Fork Skykomish River near Index, Wash.

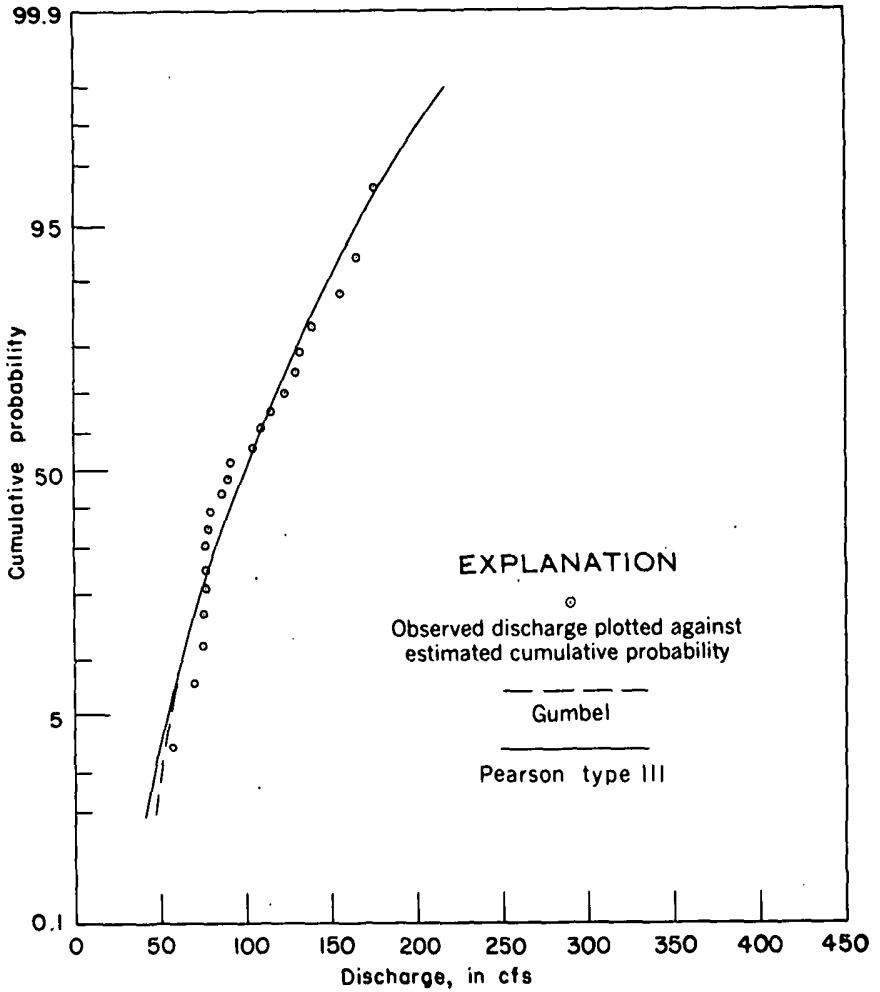

Frgure 18.-South Fork Stillaguamish River near Granite Falls, Wash.

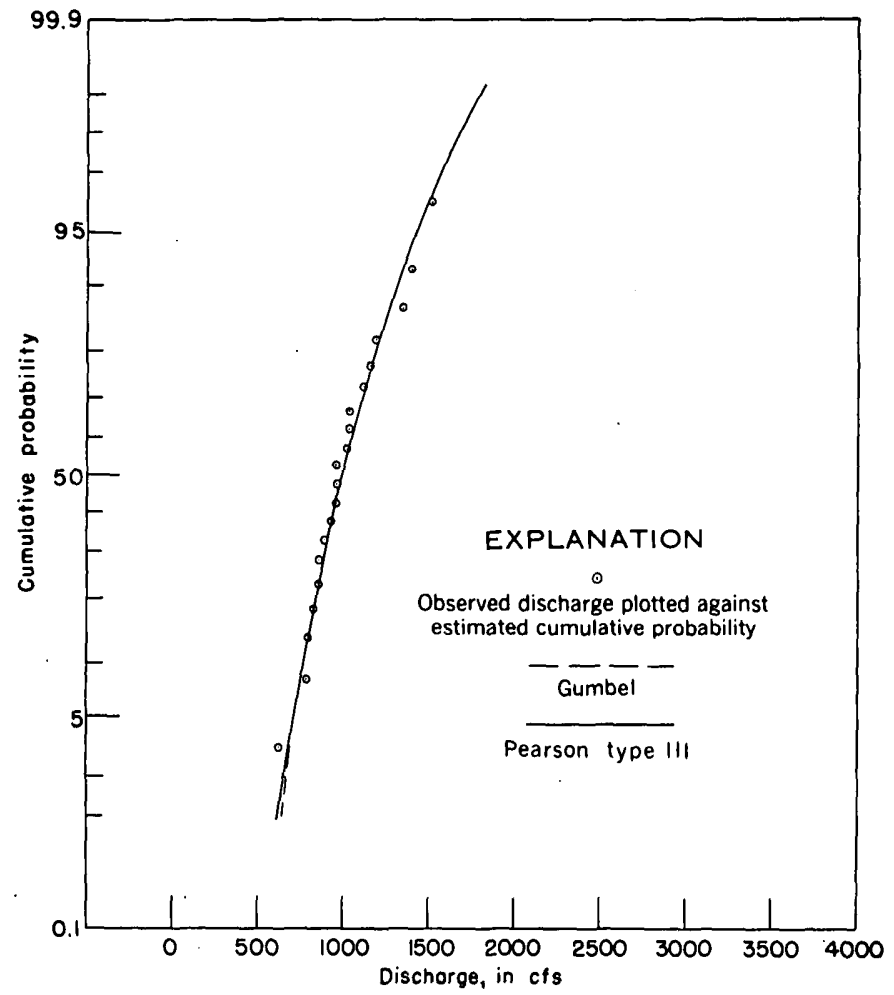

FIGORe 20.-Sauk River at Sauk, Wash. 

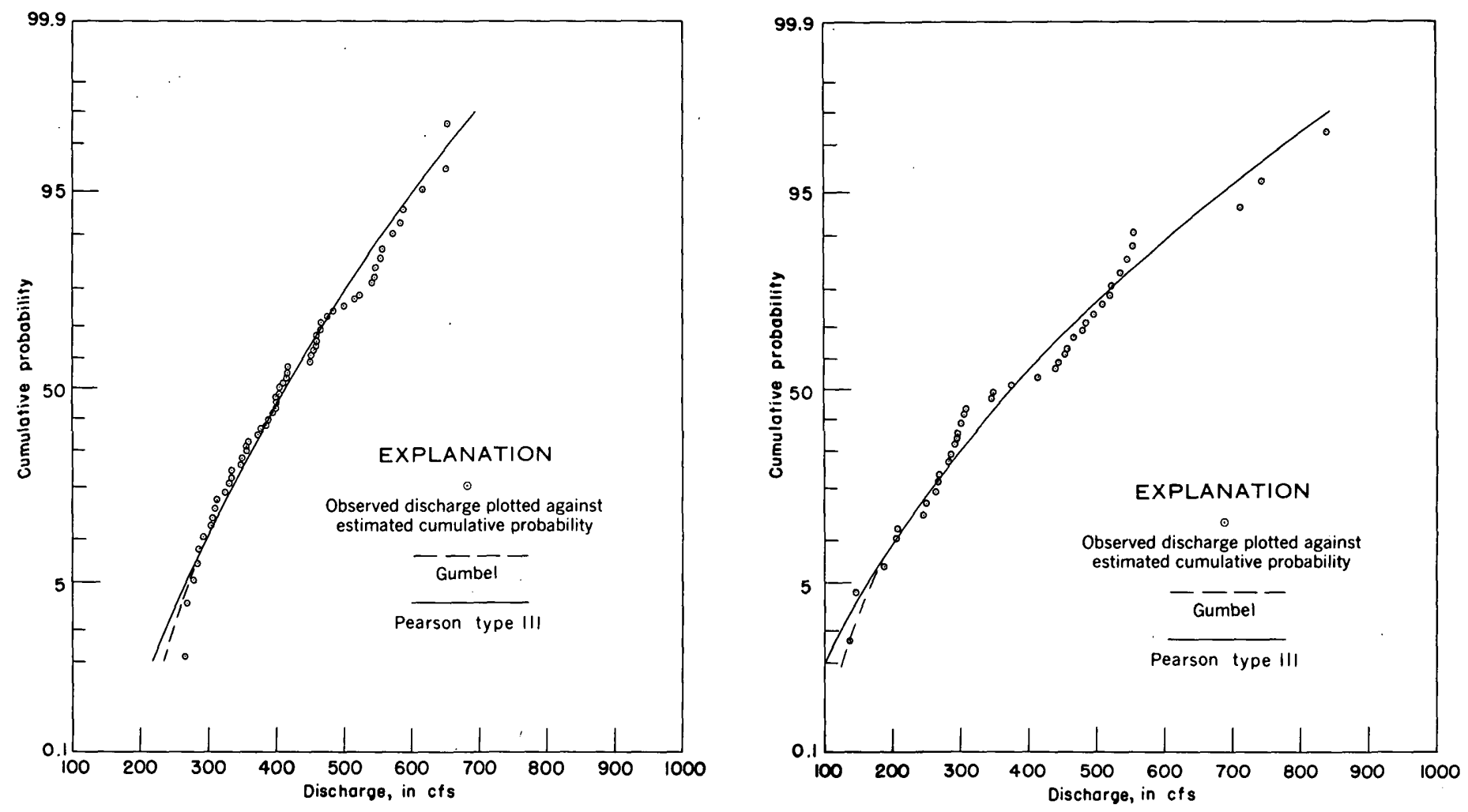

Figure 21.-James River at Buchanan, Va.

Frodre 22.--Susquehanna River at Cinklin, N.Y.
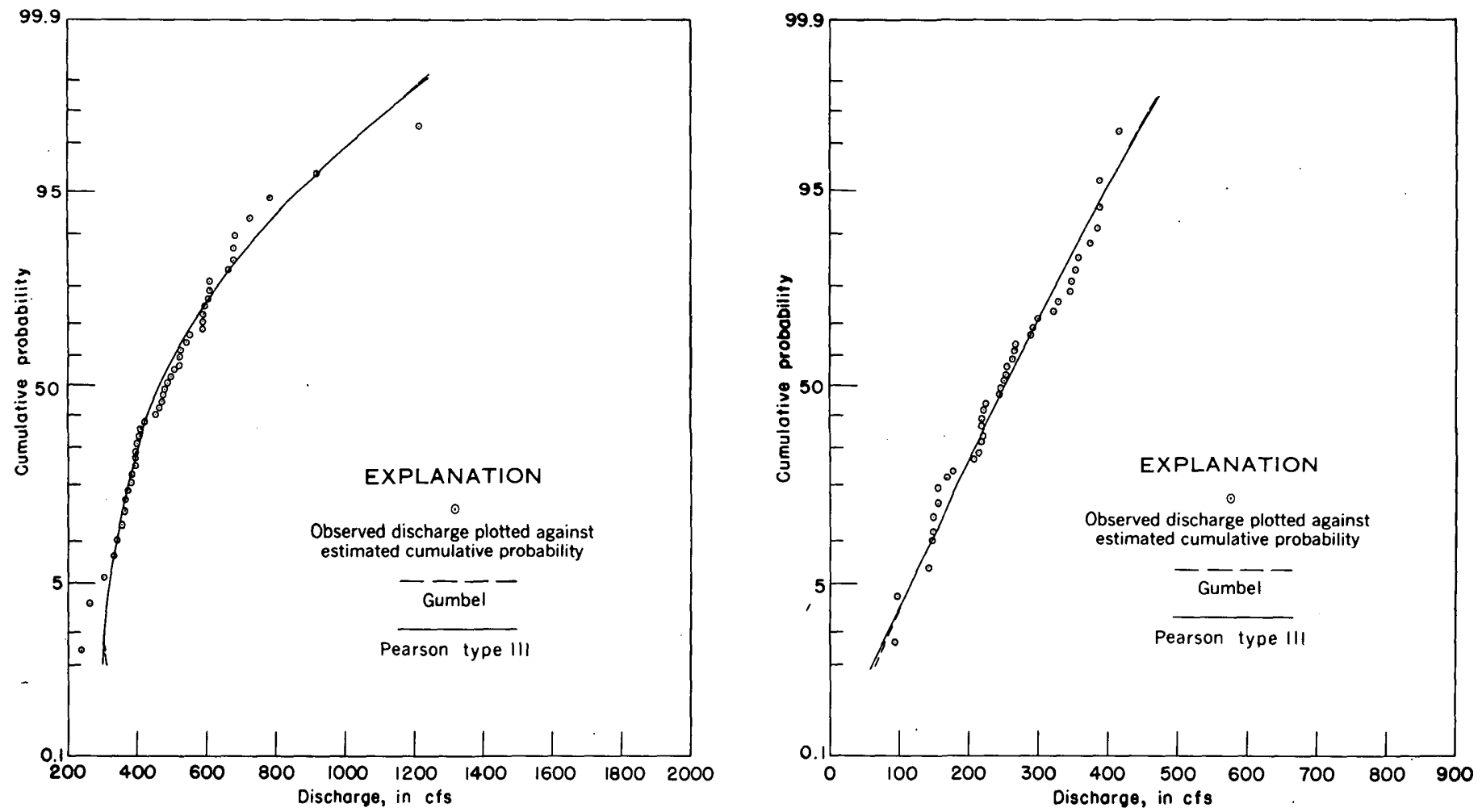

Figure 23.-Little Tennessee River at Judson, N.C.

Frouke 24.-Chenango River near Chenango, N.Y. 


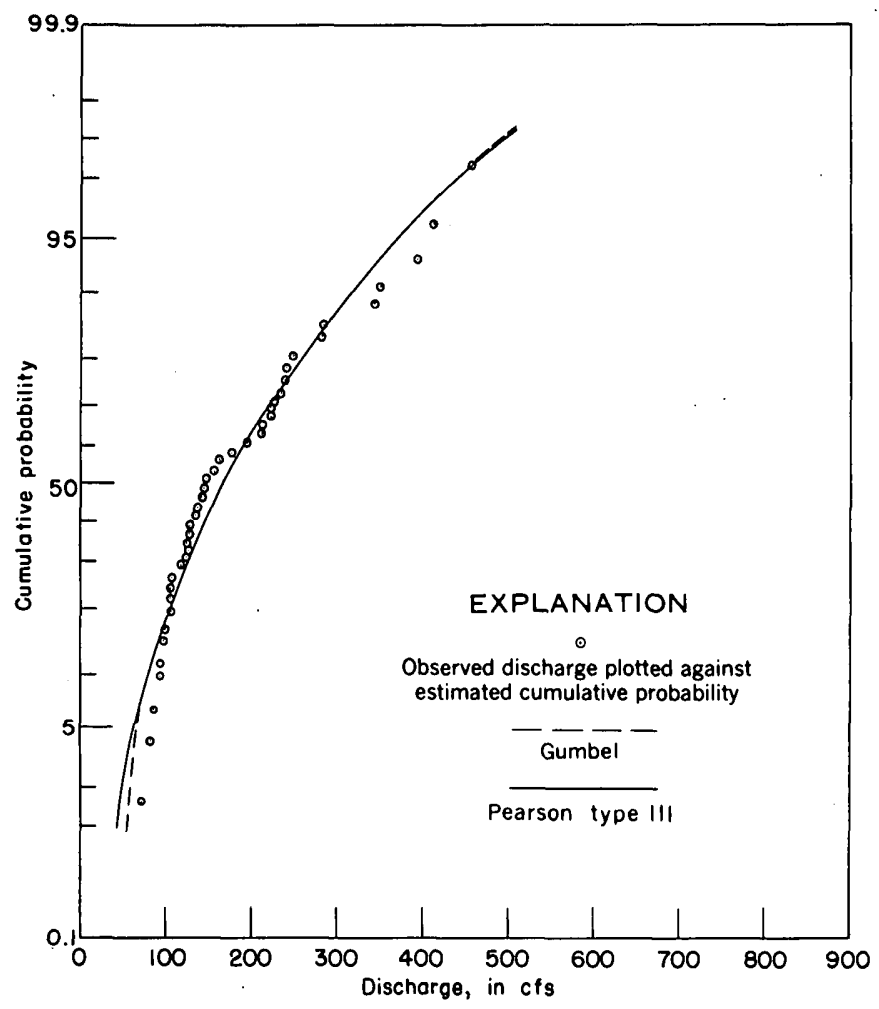

Figure 25.-East Branch Delaware River at Fish's Eddy, N.Y.

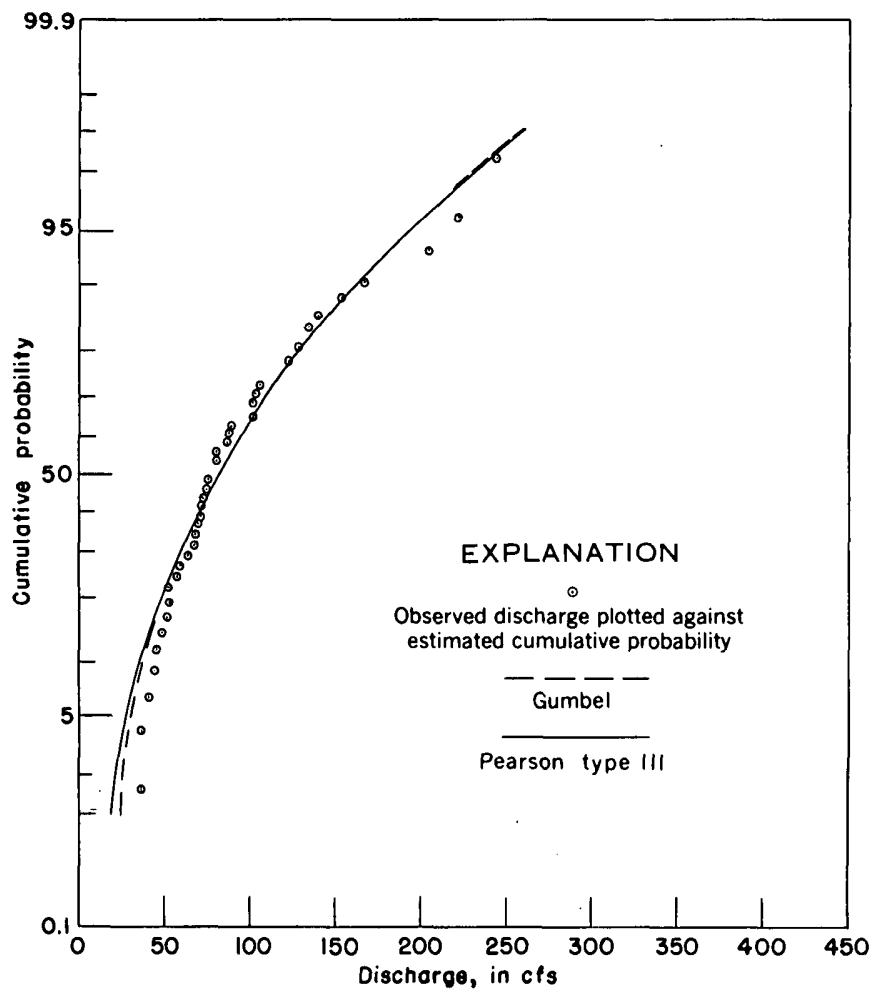

Figure 27.-Greenbrier River at Alderson, W. Va.

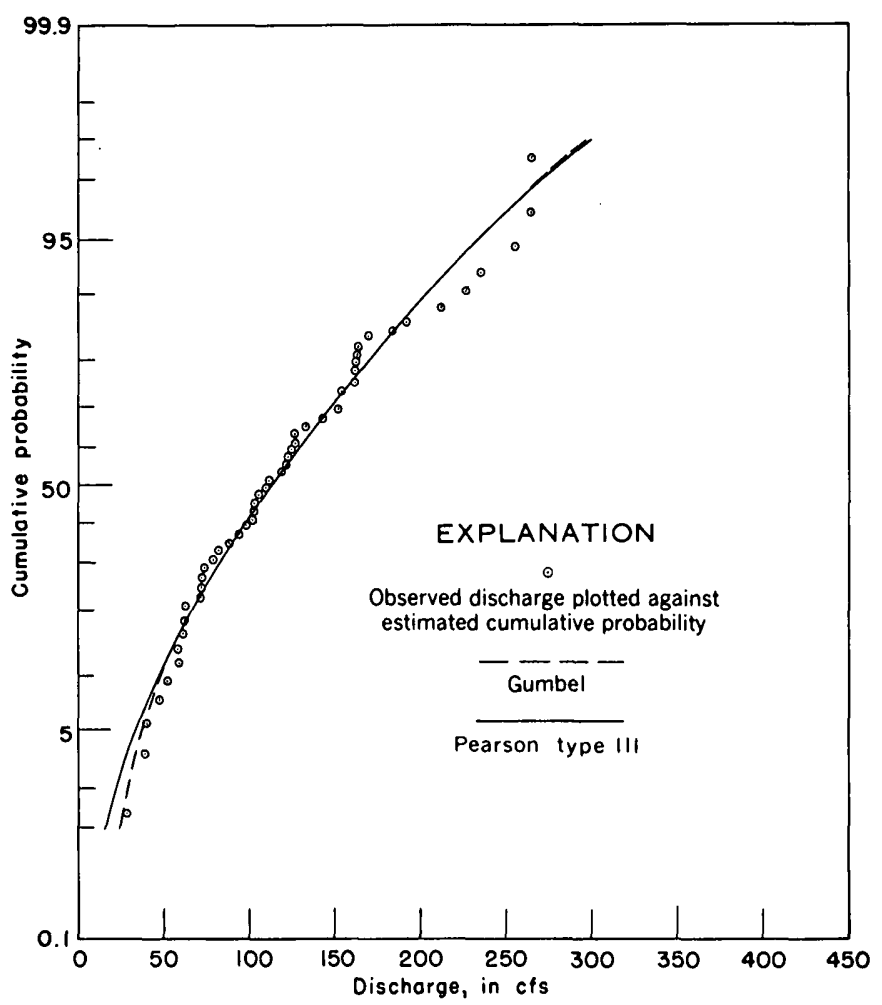

Figure 26.-West Branch Delaware River at Hales Eddy, N.Y.

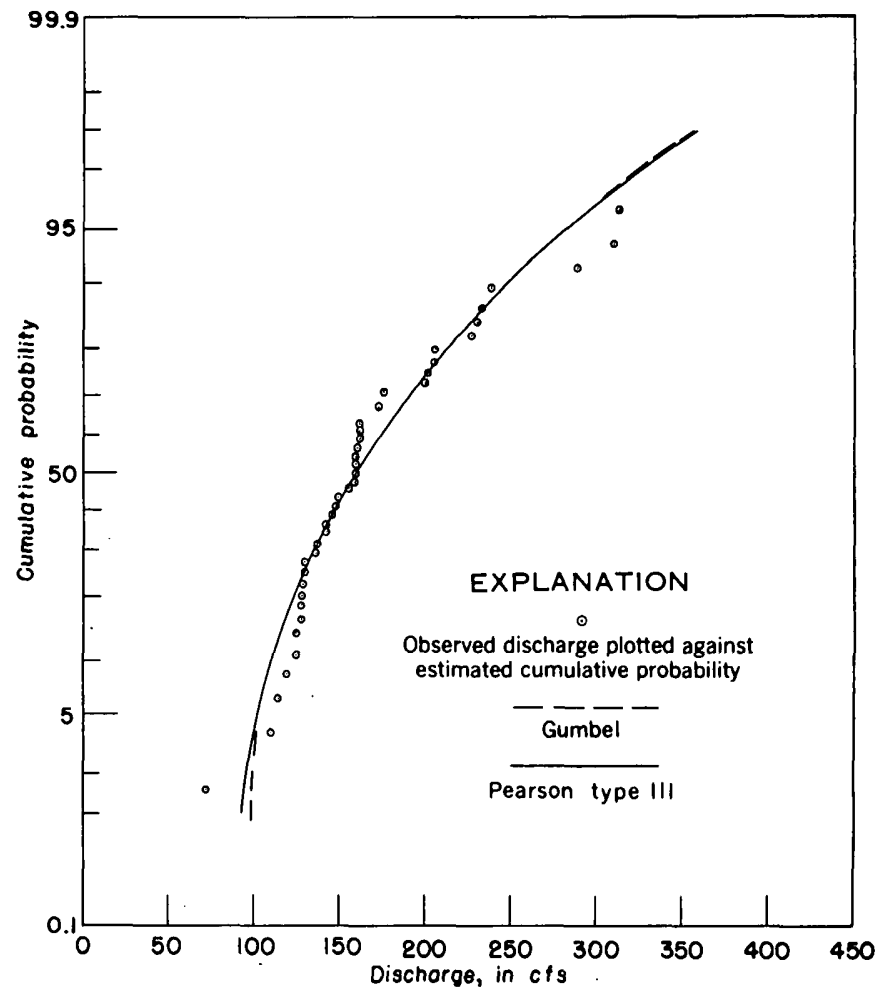

Figure 28.-Toccoa River near Dial, Ga. 


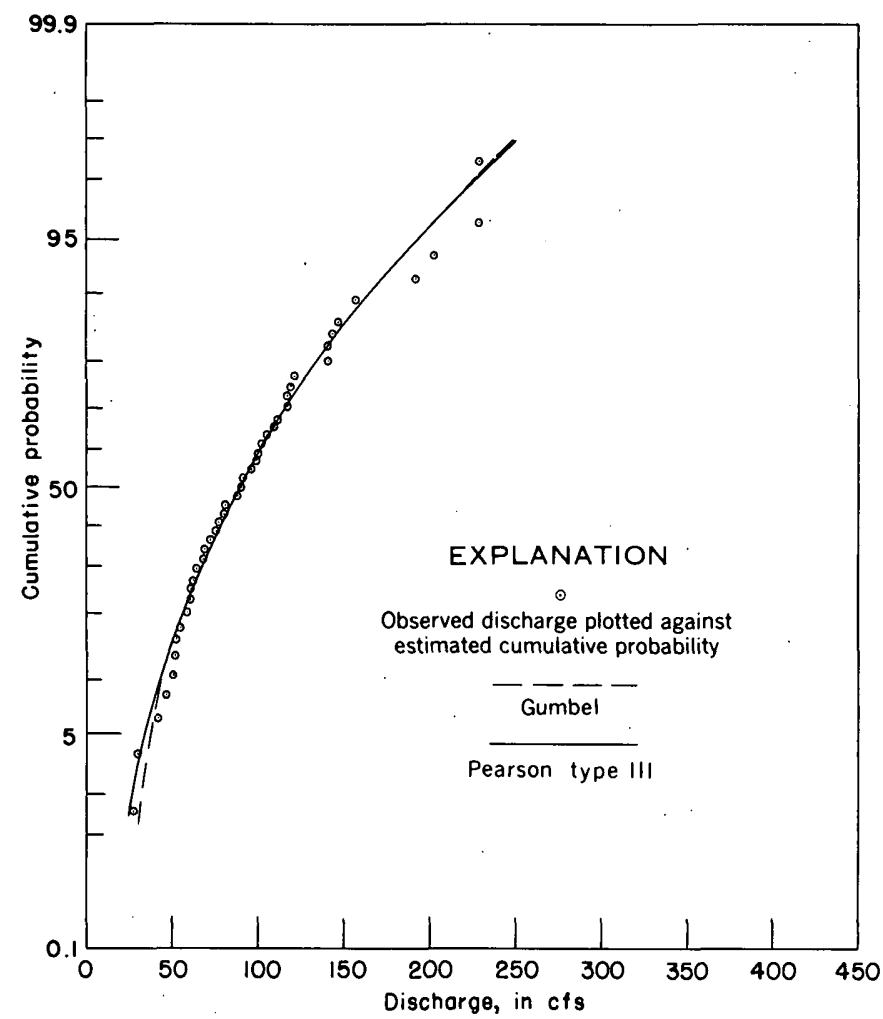

Fraure 29.-Sacandago River near Hope, N.Y.

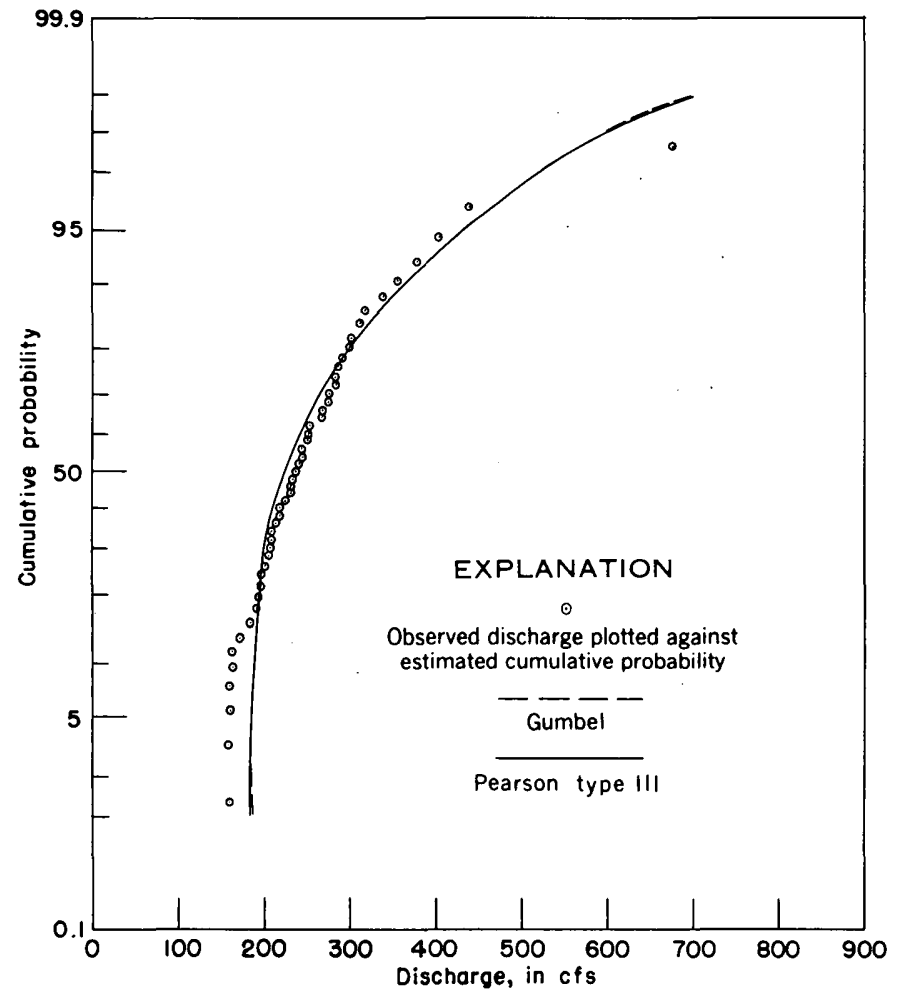

Figure 31.-South Fork of Holston River near Bluff City, Tenn.

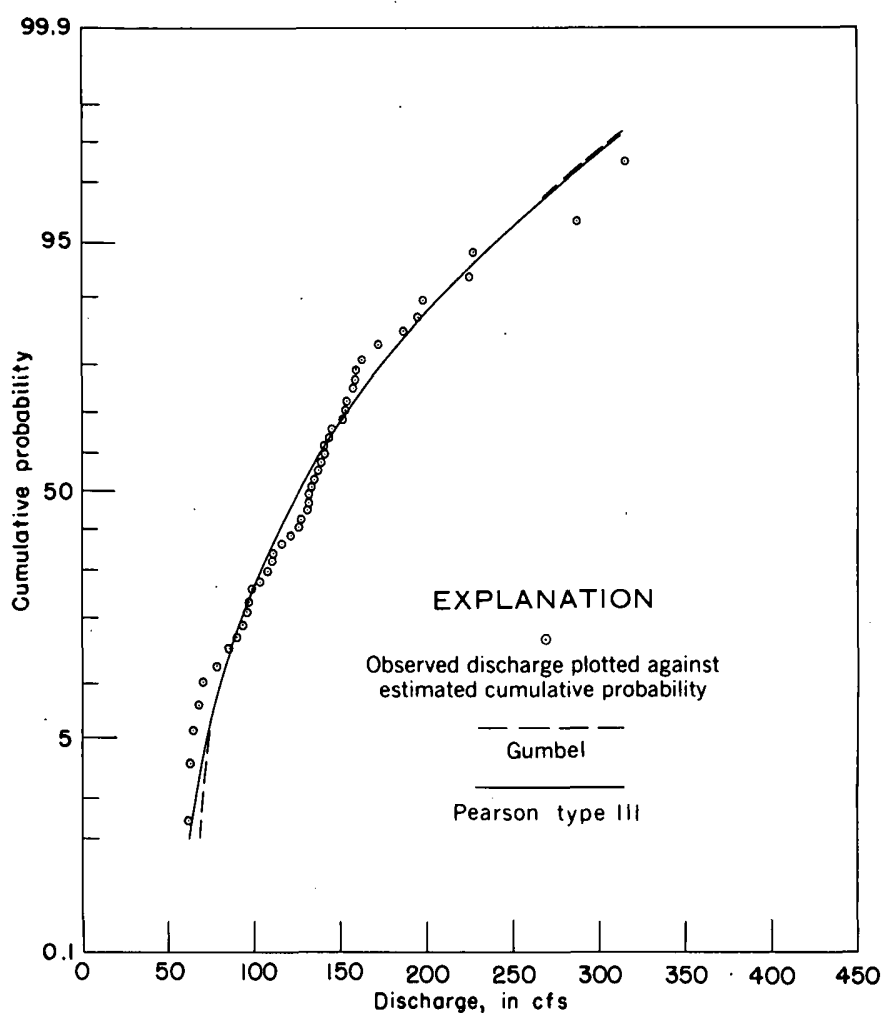

FraURe 30.-Schroon River at River Bank, N.Y.

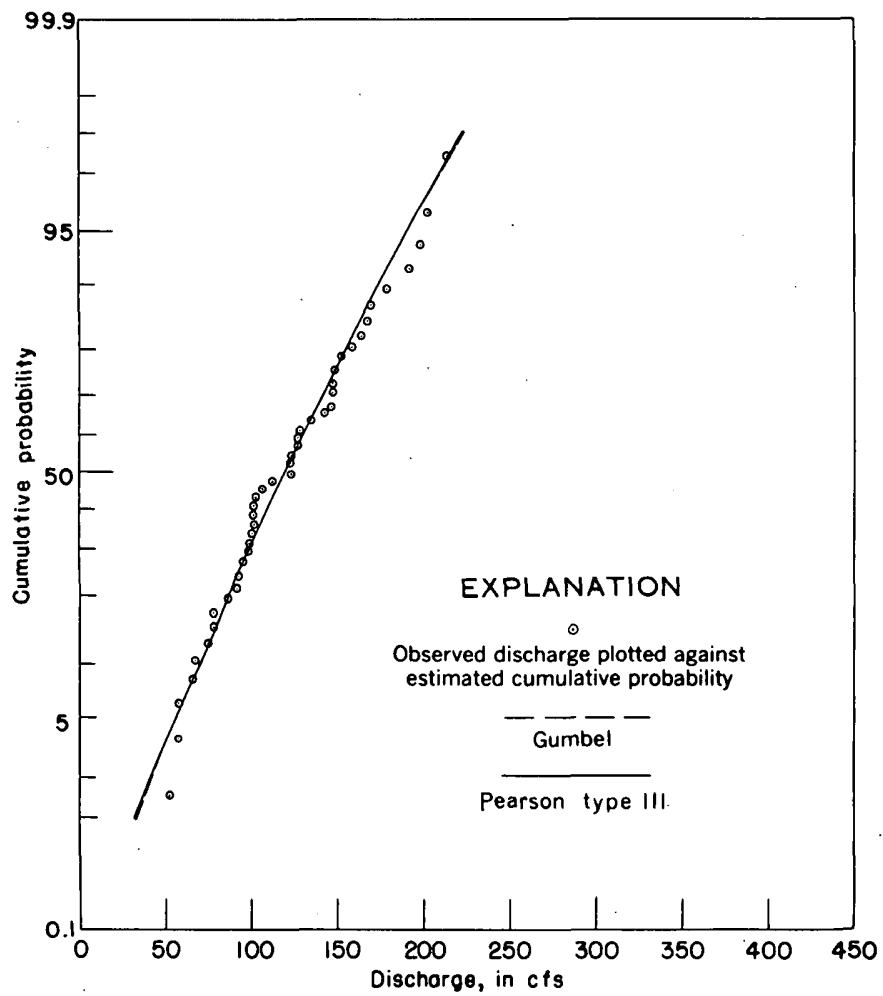

Figure 32.-Branywine River at Chadd's Ford, Pa. 


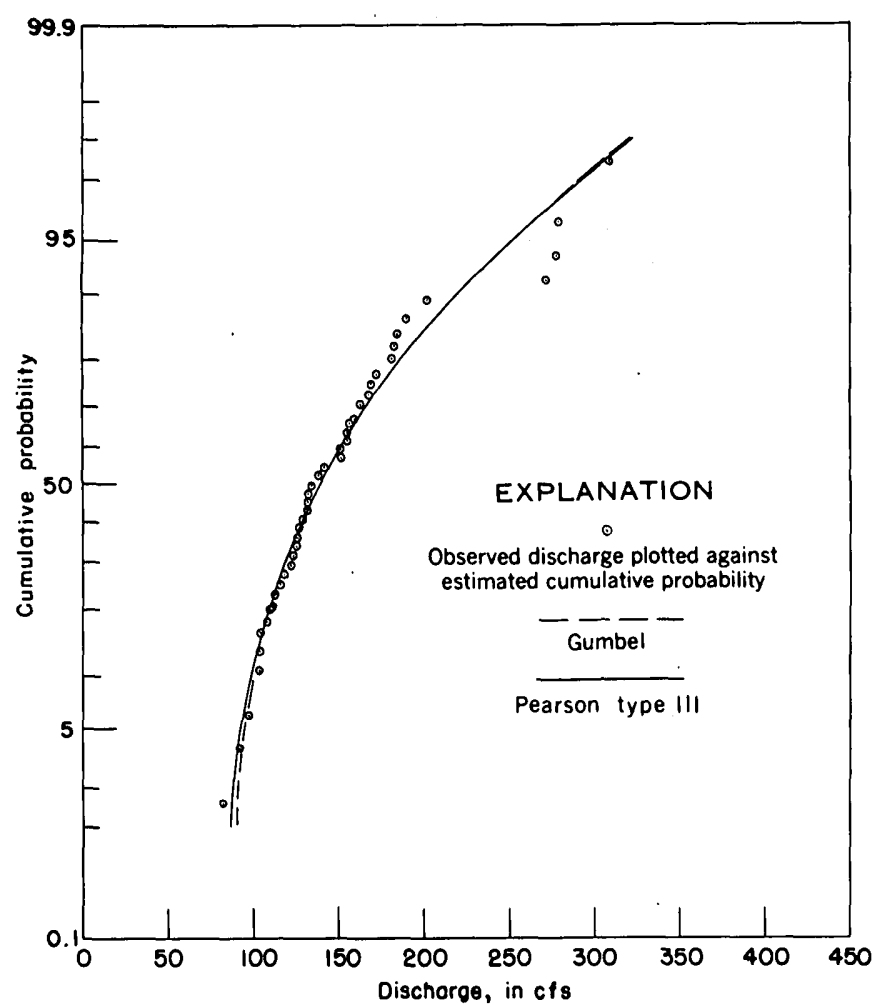

Fiaure 33.-Hoosic River near Eagle Bridge, N.Y.

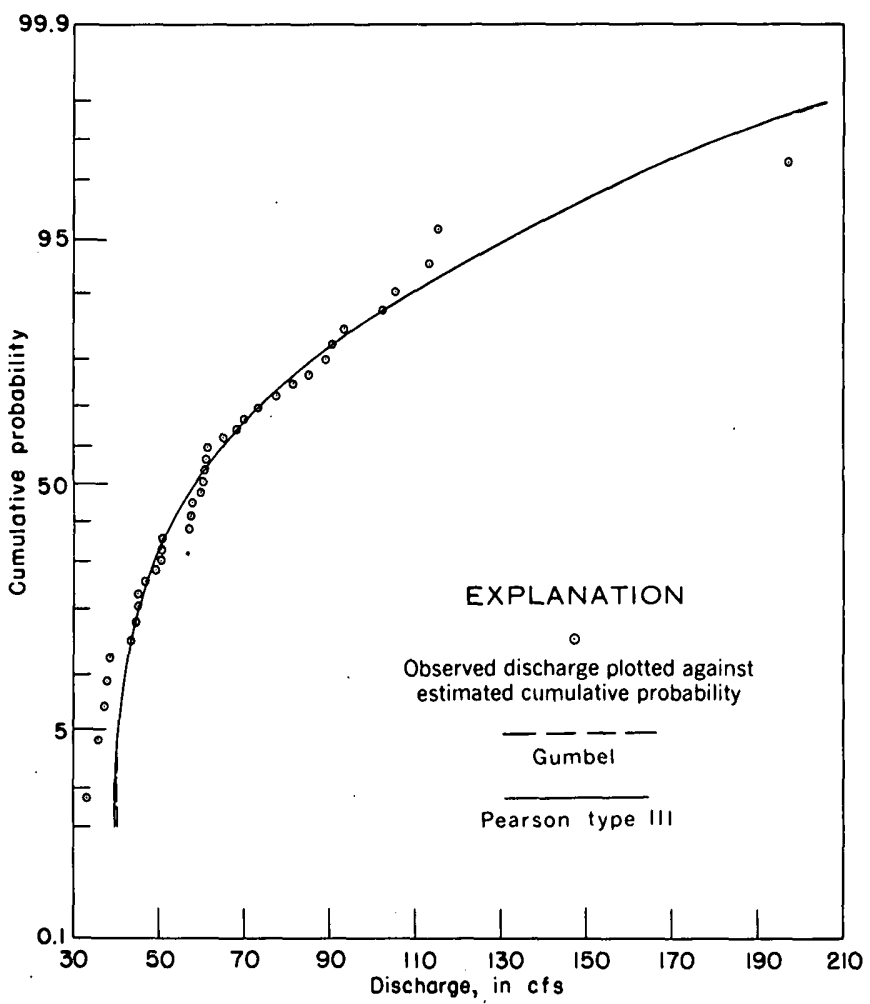

Fraure 34.-West Branch of Oswegatchie River near Harrison, N.Y.

LOW-FLOW PROBABILITY DISTRIBUTION

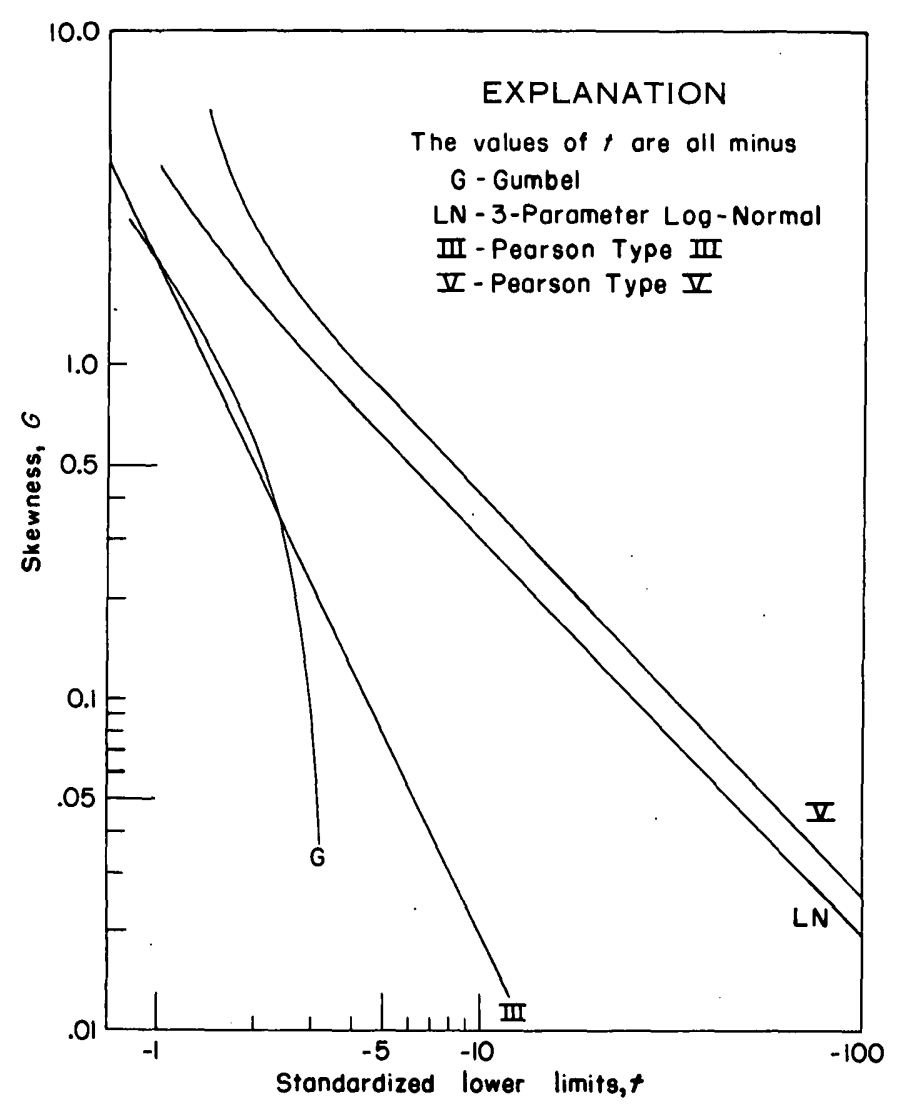

FigURE 35,-Relation between skewness and standardized lower limits for theoretical probability distributions. 


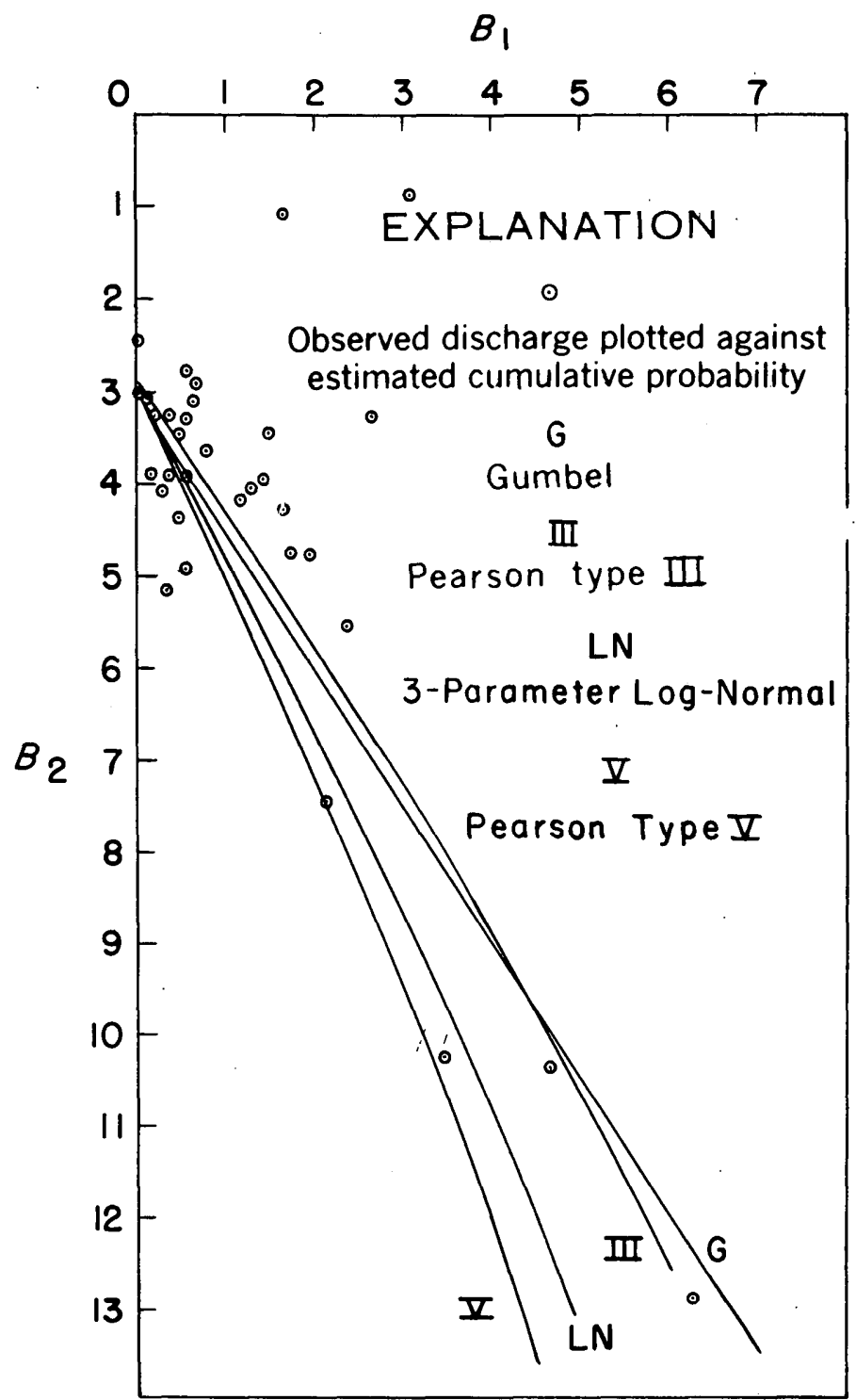

Figure 36.-Relation between skewness and kurtosis for theoretical probability distributions. 


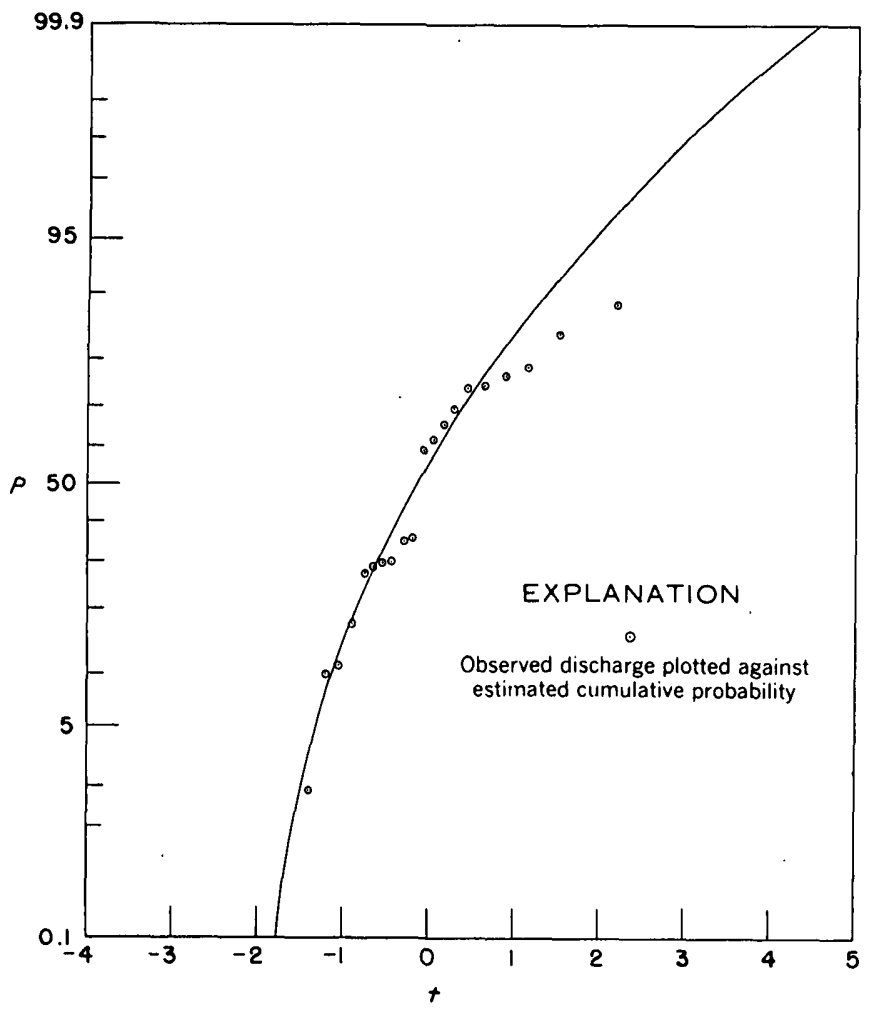

FIGURE 37. $-\mathrm{N}=20$.

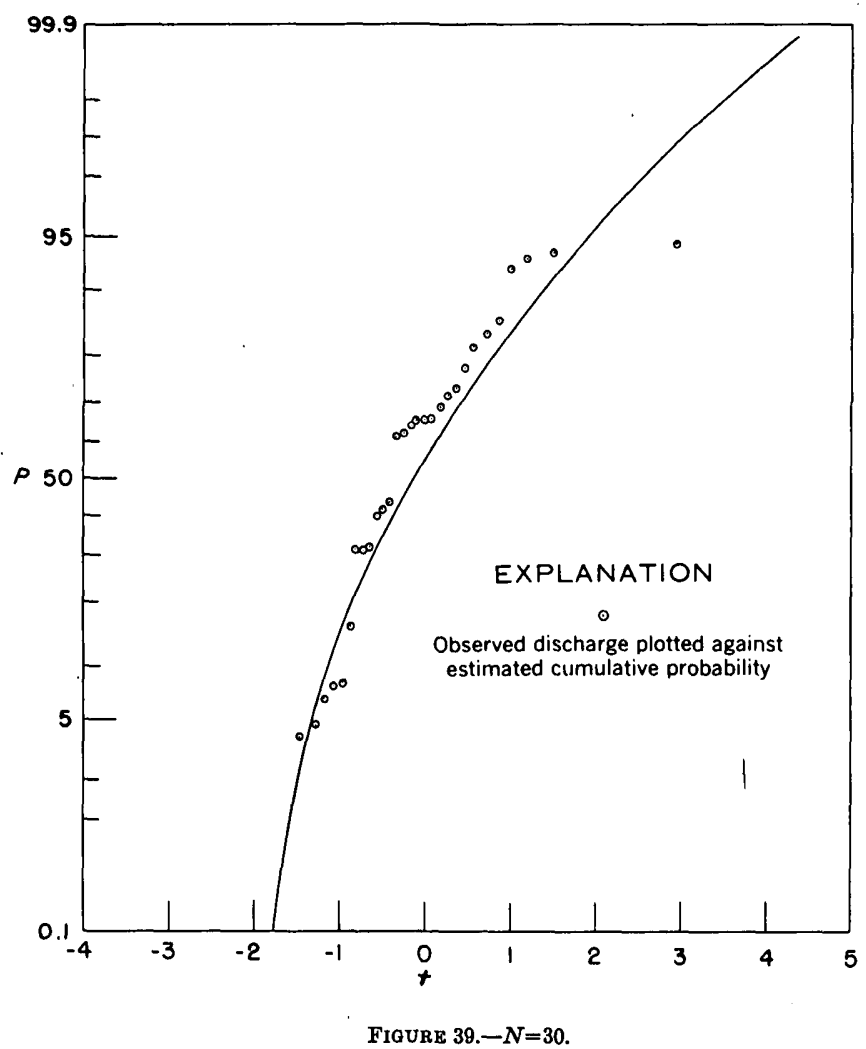

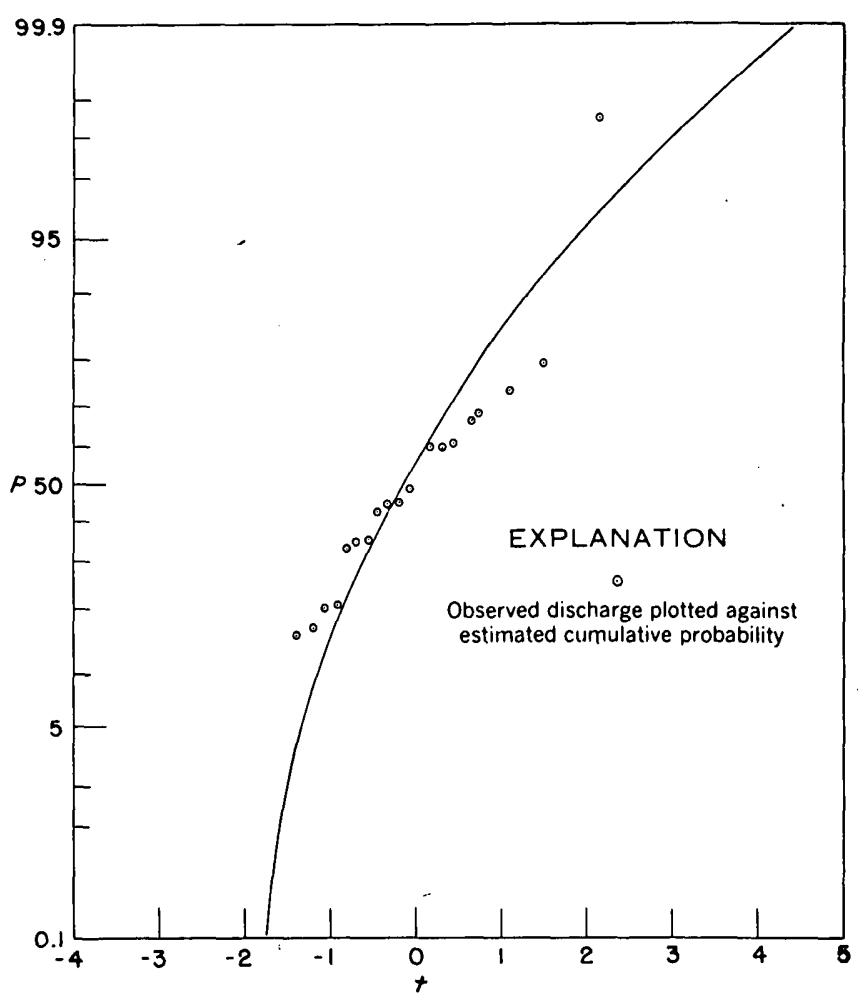

Frouge 38. $-N=20$.

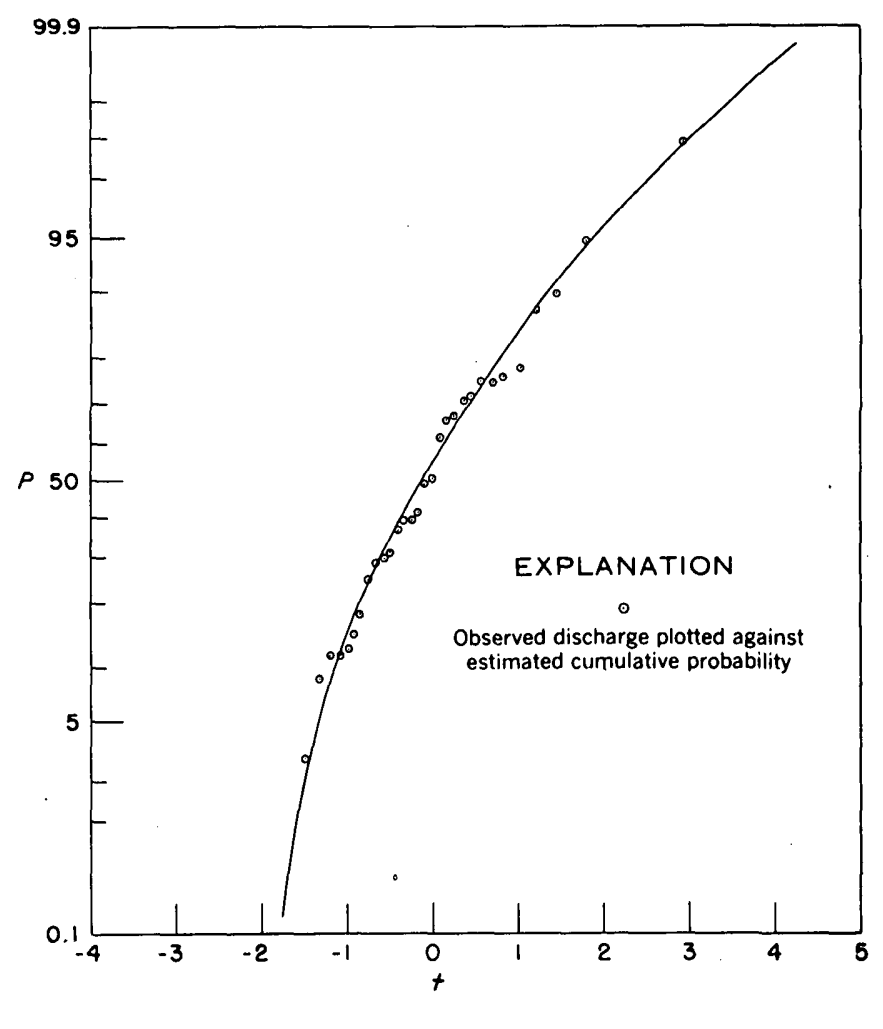

FIGURE 40. $-N=30$.

RANDOM SAMPLING FROM PEARSON TYPE III DISTRIBUTION 

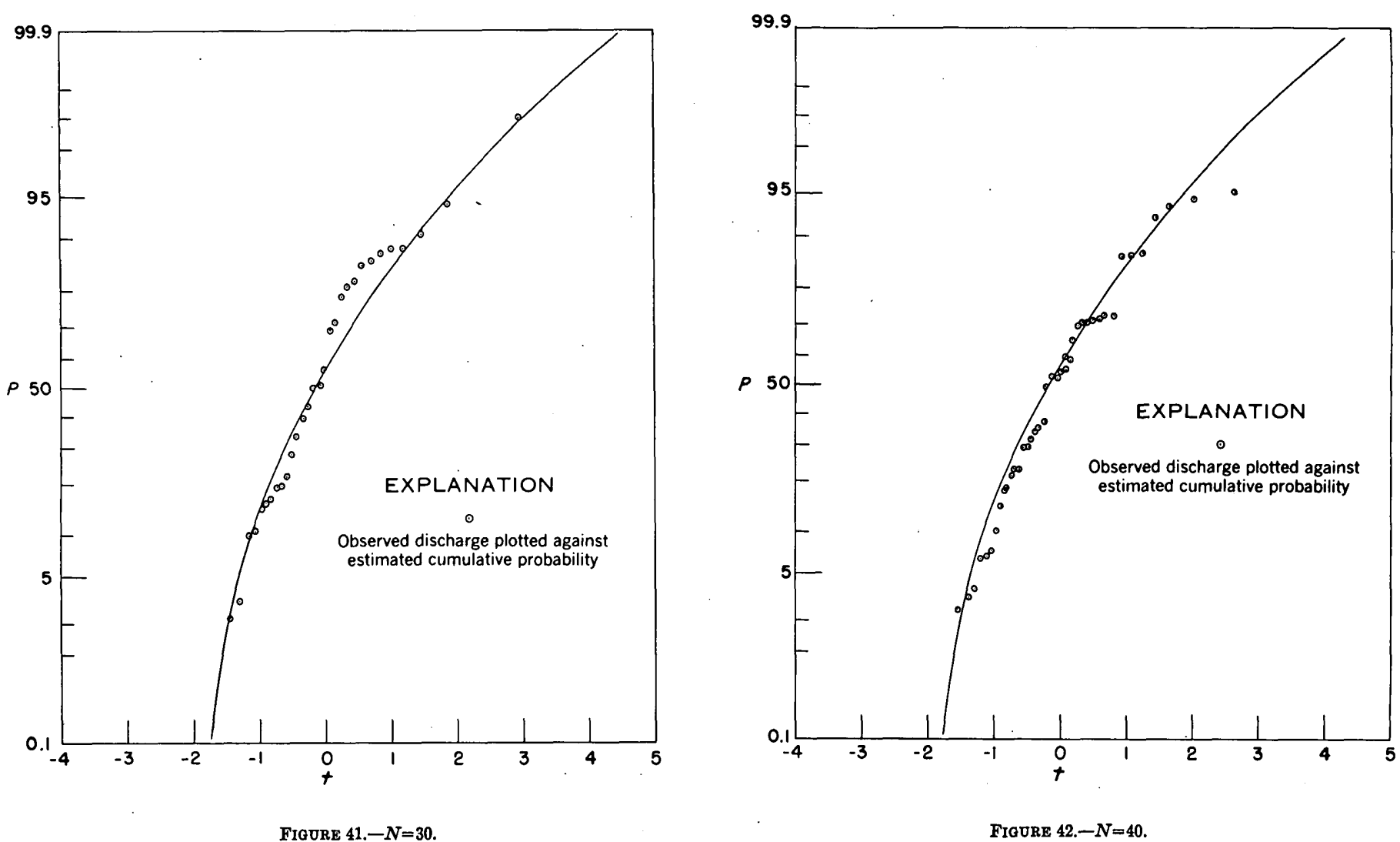

FIGURE $41 .-N=30$

FIGURE 42, $-N=40$

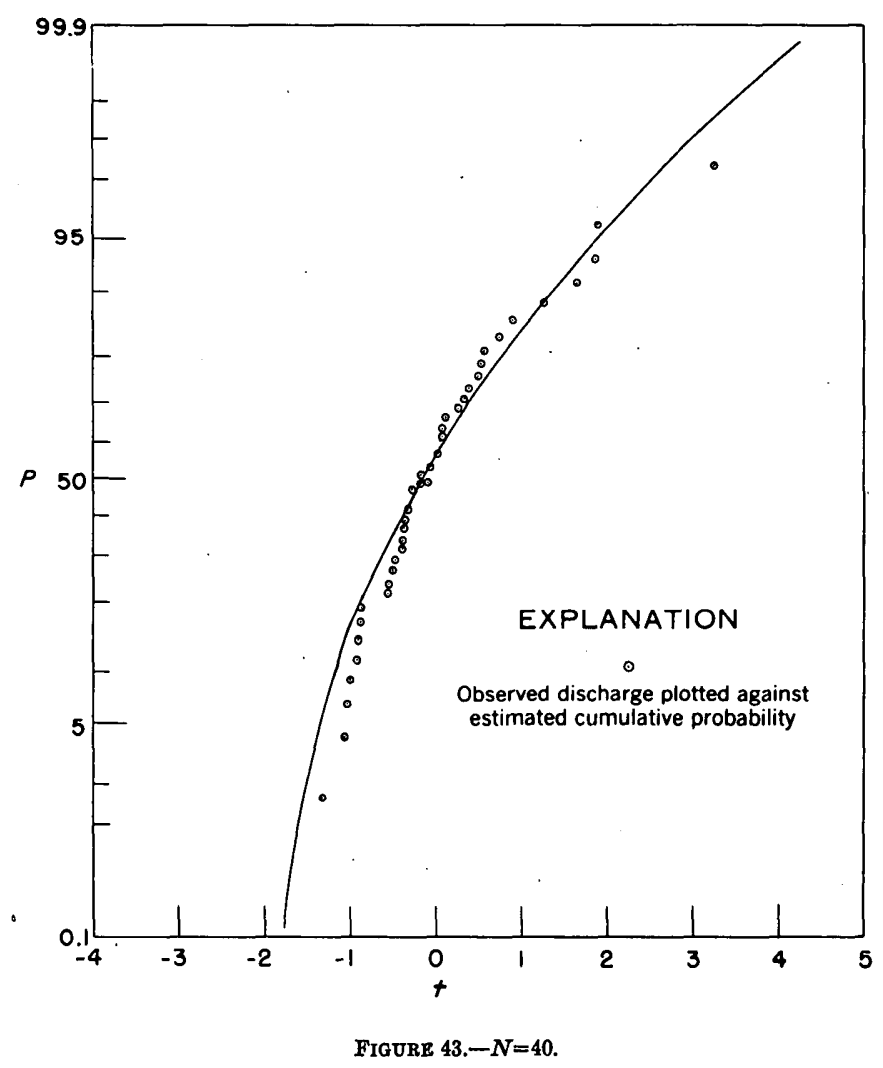

RANDOM SAMPLING FROM PEARSON TYPE III DISTRIBUTION 
REFERENCES

Fisher; R. A., 1922, On the mathematical foundations of theoretical statistics: Philos. Trans. A222, p. 309-368.

Gumbel, E. J., 1954a, Statistical theory of extreme values and some practical applications: Natl. Bur. Standards Appl. Math. Ser. 33, p. 14.
Gumbel, E. J., 1954b, Statistical theory of droughts: Am. Soc. Civil Engineers Proc., Separate 439.

Hoel, P. G., 1954; Introduction to mathematical statistics: Wiley, New York, N. Y., p. 203. 